\title{
Velocity distribution functions and intermittency in one-dimensional randomly forced Burgers turbulence
}

\author{
Victor Dotsenko \\ Sorbonne Université, LPTMC, F-75005 Paris, France and \\ L.D. Landau Institute for Theoretical Physics, 119334 Moscow, Russia
}

(Dated: May 6, 2019)

\begin{abstract}
The problem of one-dimensional randomly forced Burgers turbulence is considered in terms of $(1+1)$ directed polymers. In the limit of strong turbulence (which corresponds to the zero temperature limit for the directed polymer system) using the replica technique a general explicit expression for the joint distribution function of two velocities separated by a finite distance is derived. In particular, it is shown that at length scales much smaller than the injection length of the Burgers random force the moments of the velocity increment exhibit typical strong intermittency behavior.
\end{abstract}

PACS numbers: 05.20.-y 75.10.Nr 74.25.Qt 61.41.+e

\section{INTRODUCTION}

In the problem of one-dimensional randomly forced Burgers turbulence one studies the statistical properties a velocity field $v(x, t)$ governed by the Burgers equation [1]

$$
\partial_{t} v(x, t)+v(x, t) \partial_{x} v(x, t)=\nu \partial_{x}^{2} v(x, t)+f(x, t)
$$

where the parameter $\nu$ is the viscosity and $f(x, t)$ is the Gaussian distributed random force which is $\delta$-correlated in time and which is characterized by finite correlation length $R$ in space: $\overline{f(x, t) f\left(x^{\prime}, t^{\prime}\right)}=u \delta\left(t-t^{\prime}\right) \mathcal{F}\left[\left(x-x^{\prime}\right) / R\right]$. Here $\mathcal{F}(x)$ is a smooth function decaying to zero fast enough at large arguments and the parameter $u$ is the injected energy density. This problem is the subject of active investigations for more that six decades (see e.g. [2 [4] and references there in).

In the framework of the celebrated Kolmogorov theory [5] one obtains the probability density function (PDF) of the velocity increment $w=v\left(x_{0}+x, t\right)-v\left(x_{0}, t\right)$, such that at distances much smaller than the length scale $R$ of the random stirring force $f$, one finds simple scaling for the moments $\left\langle w^{q}\right\rangle \sim x^{\zeta(q)}$ with $\zeta(q)=n / 3$ (in particular one can prove that $\zeta(3)=1)$. This prediction is based on the assumption that the statistical properties of the velocity field is locally homogeneous, so that the corresponding PDF of $w$ depends only on $r$ and the average rate of the energy dissipation. However, extensive studies during last decades convincingly demonstrate that in fact the exponent $\zeta(q)$ significantly deviates from the Kolmogorov's law $q / 3$. The physical reason for that is the so called intermittency phenomenon, namely, formation of local coherent structures that drives a strong deviation from the mean fluctuation level of the velocity field $6-14$ ]

In the present paper using formal equivalence of the above Burger's problem, eq.(1), with the model of onedimensional directed polymers in a random potential [3] (see below) we are going to derive en explicit expression for the joint PDF $P\left(v, v^{\prime}\right)$ for two velocities separated by a distance $x$, as well as the corresponding PDF for the velocity increment $w=v^{\prime}-v$. In particular, at distances much smaller than the scale of the stirring force, $x \ll R$ this allows to demonstrate the typical intermittency behavior of the exponent $\zeta(q)$ (see Fig.1)

It is well known that the Burgers problem, eq.(1), is formally equivalent to the one of growing interfaces in a random environment described by the Kardar-Parisi-Zhang (KPZ) equation [15, 16]. Indeed, redefining

$$
v(x, t)=-\partial_{x} F(x, t)
$$

and $f(x, t)=-\partial_{x} V(x, t)$, and integrating once eq.(1), one gets the KPZ equation for the interface profile $F(x, t)$,

$$
\partial_{t} F(x, t)=\frac{1}{2}\left(\partial_{x} F(x, t)\right)^{2}+\nu \partial_{x}^{2} F(x, t)+V(x, t)
$$

where $V(x, t)$ is a random potential. On the other hand, let us consider one-dimensional directed polymers system which is defined in terms of the Hamiltonian

$$
H[\phi(\tau), V]=\int_{0}^{t} d \tau\left\{\frac{1}{2}\left[\partial_{\tau} \phi(\tau)\right]^{2}+V[\phi(\tau), \tau]\right\} ;
$$


where $\phi(\tau)$ is a scalar field defined within an interval $0 \leq \tau \leq t$ and $V(\phi, \tau)$ is the Gaussian distributed random potential with a zero mean, $\overline{V(\phi, \tau)}=0$, and the correlation function

$$
\overline{V(\phi, \tau) V\left(\phi^{\prime}, \tau^{\prime}\right)}=u \delta\left(\tau-\tau^{\prime}\right) U\left(\phi-\phi^{\prime}\right)
$$

Here the parameter $u$ defines the strength of the disorder and $U(\phi)$ is the spatial correlation function characterized by the correlation length $R$. For simplicity we take

$$
U(\phi)=\frac{1}{\sqrt{2 \pi} R} \exp \left\{-\frac{\phi^{2}}{2 R^{2}}\right\}
$$

For a given realization of the random potential $V[\phi, \tau]$ the partition function of this system is defined as

$$
Z(x, t)=\int_{\phi(0)=0}^{\phi(t)=x} \mathcal{D} \phi(\tau) \exp \{-\beta H[\phi(\tau), V]\}=\exp \{-\beta F(x, t)\}
$$

where $\beta$ is the inverse temperature, $F(x, t)$ is the free energy and the integration is taken over all trajectories $\phi(\tau)$ with the boundary conditions at $\phi(\tau=0)=0$ and $\phi(\tau=t)=x$. One can easily show that the partition function $Z(x, t)$ defined above satisfy the linear differential equation

$$
\partial_{t} Z(x, t)=\frac{1}{2 \beta} \partial_{x}^{2} Z(x, t)-\beta V(x, t) Z(x, t)
$$

Substituting here $Z(x, t)=\exp \{-\beta F(x, t)\}$, one easily finds that the free energy function $F(x, t)$ satisfy the KPZ equation (3) with the viscosity parameter $\nu=\frac{1}{2 \beta}$. In other words, the original random force Burger's problem, eq.(1) is formally equivalent the directed polymer system, eqs.(47)-(17), such that the the viscosity parameter $\nu$ in the Burger's equation is proportional to the temperature in the directed polymer system, $\nu=\frac{1}{2} T$, and the velocity $v(x, t)$ in the Burger's equation is the negative spatial derivative of the free energy $F(x, t)$ of the directed polymers system.

The standard dimensionless parameter which characterizes the level of turbulence of the velocity field in the Burgers problem is called the Reynolds number $R e$, and it is defined as the ratio of typical values of the inertial forces to viscous forces. In the present notations it can be defined as $R e=v_{0} R / \nu$, where $v_{0}$ is the typical flow velocity at the characteristic linear dimension which in the present case is the injection scale of the random force $R$. Using dimensional arguments one easily finds that

$$
v_{0} \sim\left(\frac{u}{R^{2}}\right)^{1 / 3}
$$

Indeed, according to eq.(2) the dimension of the velocity $\left[v_{0}\right]=[F] / R$. On the other hand according to eq.(44), the dimension of the free energy is $[F]=[H]=t[V]$. Finally, according to eqs.(15)-(16), the dimension of the random potential is $[V]=\sqrt{u /(R t)}$. Combining all that together one finds eq. (99). Therefore, in terms of the directed polymers notations the Reynolds number of the Burgers turbulence problem reads

$$
R e=\frac{v_{0} R}{\nu}=2 \beta(u R)^{1 / 3}
$$

It is evident that an increasing Reynolds number indicates an increasing turbulence of flow and the limit of strongly developed turbulence corresponds to $R e \rightarrow \infty$. Thus the strong turbulence Burgers regime corresponds to the zerotemperature limit in the directed polymers system and it is this limit which will be studied in the present paper.

As the velocity in the Burgers problem is given by the spatial derivative of the free energy of the directed polymer system, it can be expressed in terms of the difference of two free energies:

$$
v(x . t)=-\frac{\partial F(x, t)}{\partial x}=-\lim _{\epsilon \rightarrow 0} \frac{F(x+\epsilon, t)-F(x, t)}{\epsilon}
$$

In other words, one-point velocity statistics is defined by the joint statistics of of two free energies. Correspondingly, if we are going to study the joint statistical properties of two spatially separated velocities, in terms of the free energies of the directed polymers we have to study the four-point spatial object.

In Section II we describe the general ideas and the main lines of the replica approach which will be used in the further derivations of the probability distribution functions. In section III we describe the main points of the zerotemperature limit approach for the directed polymers with finite correlation length of the random potential, eqs. (15)-(6) 
(for details see [17]). The zero temperature limit of the joint probability distribution function of free energies defined at four spatial points is derived in Section IV. The explicit expression for the corresponding joint probability density function of two velocities $v$ and $v^{\prime}$ separated by a distance $x$ is derived in Section V, eqs.(111)-(113). In Section VI it will be shown that the PDF for the velocity increment $w=v-v^{\prime}$ has the following form:

$$
P_{x}(w)=p_{0}(x / R) \delta\left(w-v_{0} \frac{x}{R}\right)+\mathcal{P}_{x / R}\left(w / v_{0}\right) \theta\left(v_{0} \frac{x}{R}-w\right)
$$

where $\theta(z)$ is the Heaviside step function, $v_{0} \propto\left(u / R^{2}\right)^{1 / 3}$ (see eq. (9)),

$$
p_{0}(x / R)=\int_{-\infty}^{+\infty} \frac{d s}{\sqrt{2 \pi}} \frac{\exp \left\{-\frac{1}{2} s^{2}\right\}}{\left(1+\frac{\zeta_{0}^{3 / 4} x}{R} \int_{0}^{+\infty} \frac{d \xi}{\sqrt{2 \pi}} \xi \exp \left\{-\frac{1}{2}(s+\xi)^{2}\right\}\right)}
$$

and

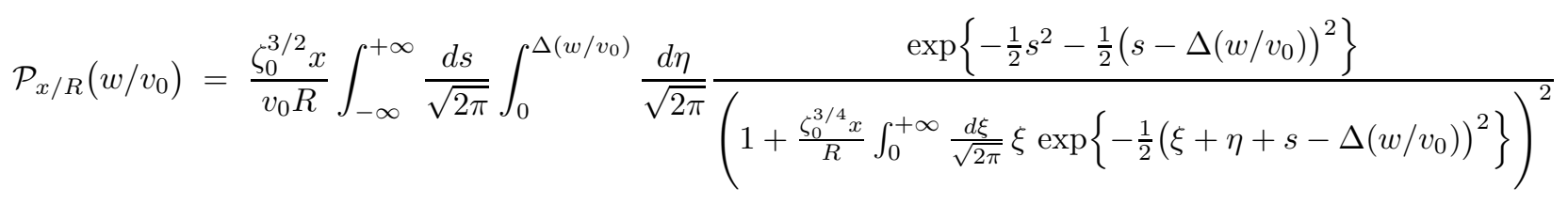

Here $\zeta_{0} \sim 1$ is a number (see Section III) and

$$
\Delta\left(w / v_{0}\right)=\zeta_{0}^{3 / 4}\left(\frac{x}{R}-\frac{w}{v_{0}}\right)
$$

The above formulas, eqs.(12)-(15) constitute the central result of the present research. The distribution function $P_{x}(w)$ has rather specific structure (see Section VI, Fig.4). According to eq. (12) for a given distance $x$ the values of the velocity increment $w$ are bounded from above: $w \leq \frac{x}{R} v_{0}$, where $v_{0} \propto\left(u / R^{2}\right)^{1 / 3}$ is the typical flow velocity at the injection scale $R$ of the random force of the strength $u$. Moreover, at $w=\frac{x}{R} v_{0}$ the distribution function exhibits the $\delta$-function singularity which means that at a given distance $x$ the difference of two velocities $w=v-v^{\prime}$ has a finite probability $p_{0}$, eq.(13), to be equal to $\frac{x}{R} v_{0}$.

The above result allows to study the behavior of the moments of the velocity increment $\left\langle w^{q}\right\rangle$ at distances $x \ll R$. Introducing the reduced distance parameter $r=\zeta_{0}^{3 / 4} x / R$ and the reduced velocity increment $\omega=\zeta_{0}^{3 / 4} w / v_{0}$, in the limit $r \ll 1$ instead of eqs.(12)-(14) we get:

$$
P_{r}(\omega) \simeq\left(1-\frac{r}{\sqrt{\pi}}\right) \delta(\omega-r)+\frac{r}{\sqrt{\pi}}(r-\omega) \exp \left\{-\frac{1}{4}(r-\omega)^{2}\right\} \theta(r-\omega)
$$

Then, for even moments of the reduced velocity increment we obtain:

$$
\left\langle\omega^{2 n}\right\rangle \simeq r^{2 n}+C(n) r
$$

where $C(n)=2^{2 n+1} \Gamma(1+n)$. The above result can be analytically continued for arbitrary real values $q$ of the parameter $2 n \rightarrow q$. Then, introducing the exponent $\zeta(q)$ as $\left\langle\omega^{q}\right\rangle \simeq r^{\zeta(q)}$, according to eq. (17) in the limit $r \ll 1$ we recover the typical strong intermittency behavior (see Fig.1):

$$
\zeta(q) \simeq\left\{\begin{array}{l}
q, \text { for } q \leq 1 \\
1, \text { for } q>1
\end{array}\right.
$$

It should be stressed that the above results, eqs.(12) and (18), are in remarkable agreement with the ones obtained for the same system many years ago in [3] in the framework of the Gaussian variation method (which formally should be valid only at high dimensions).

\section{REPLICA FORMALISM}

In this section we are going to describe the general scheme of calculations of the statistical properties of the Burgers velocity field $v(x, t)$, eq.(11), in terms of the standard replica approach used for the directed polymers model, eqs.(4)(17). Using the relation between $v(x, t)$ and the free energy $F(x, t)$ of the corresponding directed polymer model, 


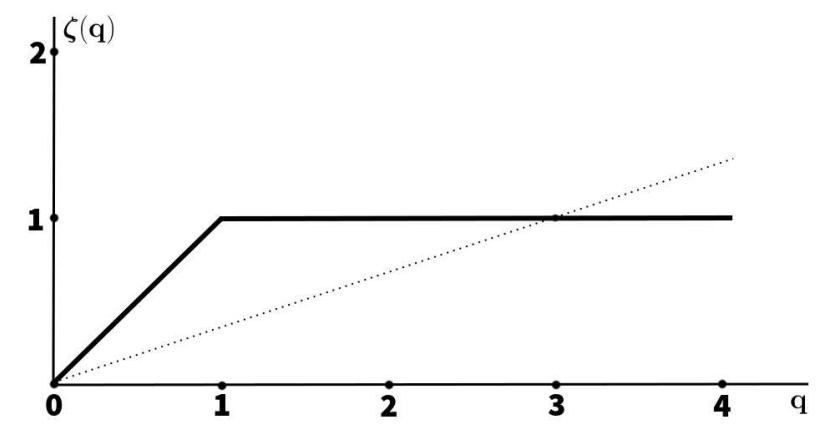

FIG. 1: Intermittency behavior of the exponent $\zeta(q)$, eq. (18). The dashed line represents the Kolmogorov scaling $\zeta(q)=q / 3$

eq.(11), for finite value of the parameter $\epsilon$ (which should be taken to zero in the final result) we have

$$
\exp \{\beta \epsilon v(x, t)\}=\exp \{-\beta F(x+\epsilon, t)+\beta F(x, t)\}=Z(x+\epsilon, t) \cdot Z^{-1}(x, t)
$$

Taking the integer power $N$ of both sides of the above relation and averaging over the disorder (which in what follows will be denoted by the overline, $\overline{(\ldots)})$ we gets

$$
\int d v P_{x, \epsilon, t}(v) \exp \{\beta N \epsilon v\}=\overline{Z^{N}(x+\epsilon, t) \cdot Z^{-N}(x, t)}
$$

where $P_{x, \epsilon, t}(v)$ is the PDF of the velocity $v$. Formally, the above relation can be represented as follows,

$$
\int d v P_{x, \epsilon, t}(v) \exp \{\beta N \epsilon v\}=\lim _{M \rightarrow 0} Z(M, N, x, \epsilon, t)
$$

where

$$
Z(M, N, x, \epsilon, t) \equiv \overline{Z^{N}(x+\epsilon, t) \cdot Z^{M-N}(x, t)}
$$

is the two-point replica partition function.

General scheme of calculations of the velocity PDF defined by the relation (21) consists of several steps. First, for a given (finite) $\epsilon$ and integers $M$ and $N$, such that $M>N$, one has to compute the replica partition function $Z(M, N, x, \epsilon, t)$ as an analytic function of the parameters $M$ and $N$. Next, this function should be analytically continued for arbitrary complex values of $M$ and $N$, and the limits $M \rightarrow 0$ as well as $t \rightarrow \infty$ have to be taken. Next, to take the limit $\epsilon \rightarrow 0$, one introduces the parameter $s=\beta \epsilon N$ which has to be kept finite (this implies that together with the limit $\epsilon \rightarrow 0$ one simultaneously takes the limit $N \rightarrow \infty$ ). Thus, after performing these manipulations (provided all the above limits exist) the relation (21) turns into bilateral Laplace transform for the velocity PDF $P_{*}(v)=\lim _{\epsilon \rightarrow 0} \lim _{t \rightarrow \infty} P_{x, \epsilon, t}(v)$ (which for finite values of $x$ in the limit $t \rightarrow \infty$ should be $x$-independent):

$$
\int d v P_{*}(v) \exp \{s v\}=Z_{*}(s)
$$

where

$$
Z_{*}(s)=\lim _{\epsilon \rightarrow 0} \lim _{t \rightarrow \infty} \lim _{M \rightarrow 0} Z\left(M, \frac{s}{\beta \epsilon}, x, \epsilon, t\right)
$$

In this way the PDF $P_{*}(v)$ could be recovered by the inverse Laplace transform.

Note that the above type of program has been already successfully implemented for the derivation of the Burgers two-point velocity PDF in the toy (Gaussian) Larking model of random directed polymers [18].

In this paper we are going to derive joint PDF of two velocities $v=v(x / 2, t)$ and $v^{\prime}=v(-x / 2, t)$ at two points separated by a finite distance $x$. In this case straightforward generalization of the above replica scheme would require computation of the four-point replica partition function:

$$
\int d v d v^{\prime} P_{x, \epsilon, t}\left(v, v^{\prime}\right) \exp \left\{\beta N_{1} \epsilon v+\beta N_{2} \epsilon v^{\prime}\right\}=\lim _{M \rightarrow 0} \overline{Z^{N_{1}}(x / 2, t) Z^{M-N_{1}}(x / 2-\epsilon, t) Z^{N_{2}}(-x / 2+\epsilon, t) Z^{M-N_{2}}(-x / 2, t)}
$$


Technically, direct recovery (using the inverse Laplace transformation) of the two-velocity PDF using the above relation turns out to be rather involved task which still remains to be done. On the other hand, the experience shows that sometimes to compute a complicated quantity, first one just has to compute a more general object. In the present case, instead of two velocities PDF let us consider the joint distribution function of three free energy differences. Namely, for a given four spatial points, $-x / 2,-x / 2+\epsilon, x / 2-\epsilon$ and $x / 2$, let us define

$$
\begin{aligned}
& f_{1}=F(x / 2, t)-F(-x / 2, t) \\
& f_{2}=F(x / 2-\epsilon, t)-F(-x / 2, t) \\
& f_{3}=F(-x / 2+\epsilon, t)-F(-x / 2, t)
\end{aligned}
$$

In terms of the partition functions the above relations can be represented as follows:

$$
\begin{aligned}
Z(x / 2, t) Z^{-1}(-x / 2, t) & =\exp \left\{-\beta f_{1}\right\} \\
Z(x / 2-\epsilon, t) Z^{-1}(-x / 2, t) & =\exp \left\{-\beta f_{2}\right\} \\
Z(-x / 2+\epsilon, t) Z^{-1}(-x / 2, t) & =\exp \left\{-\beta f_{3}\right\}
\end{aligned}
$$

As the further considerations will be done in the zero temperature limit (which correspond to the limit of large Reynolds number, eq.(10) ), it turns out that the simplest way to derive the joint PDF $P_{x, \epsilon, t}\left(f_{1}, f_{2}, f_{3}\right)$ is to use the generating function approach. Namely, let us introduce the probability function

$$
W_{x, \epsilon, t}\left(f_{1}, f_{2}, f_{3}\right)=\int_{-\infty}^{f_{1}} d f_{1}^{\prime} \int_{-\infty}^{f_{2}} d f_{2}^{\prime} \int_{-\infty}^{f_{3}} d f_{3}^{\prime} P_{x, \epsilon, t}\left(f_{1}^{\prime}, f_{2}^{\prime}, f_{3}^{\prime}\right)
$$

One can easily see that in the zero temperature limit this function can be represented in a form of the series:

$$
\begin{aligned}
& W_{x, \epsilon, t}\left(f_{1}, f_{2}, f_{3}\right)=-\lim _{\beta \rightarrow \infty} \sum_{N_{1}=1}^{\infty} \frac{(-1)^{N_{1}}}{N_{1} !} \sum_{N_{2}=1}^{\infty} \frac{(-1)^{N_{2}}}{N_{2} !} \sum_{N_{3}=1}^{\infty} \frac{(-1)^{N_{3}}}{N_{3} !} \exp \left\{\beta N_{1} f_{1}+\beta N_{2} f_{2}+\beta N_{3} f_{3}\right\} \times \\
& \times[(29) \\
& {\left[Z(x / 2, t) Z^{-1}(-x / 2, t)\right]^{N_{1}}\left[Z(x / 2-\epsilon, t) Z^{-1}(-x / 2, t)\right]^{N_{2}}\left[Z(-x / 2+\epsilon, t) Z^{-1}(-x / 2, t)\right]^{N_{3}} }
\end{aligned}
$$

Indeed, substituting here eq.(27) we gets

$$
\begin{aligned}
W_{x, \epsilon, t}\left(f_{1}, f_{2}, f_{3}\right) & =-\lim _{\beta \rightarrow \infty} \int_{-\infty}^{+\infty} d f_{1}^{\prime} \int_{-\infty}^{+\infty} d f_{2}^{\prime} \int_{-\infty}^{+\infty} d f_{3}^{\prime} P_{x, \epsilon, t}\left(f_{1}^{\prime}, f_{2}^{\prime}, f_{3}^{\prime}\right)\left[\sum_{N_{1}=1}^{\infty} \frac{(-1)^{N_{1}}}{N_{1} !} \exp \left\{\beta\left(f_{1}-f_{1}^{\prime}\right) N_{1}\right\}\right] \times \\
& \left.\times\left[\sum_{N_{2}=1}^{\infty} \frac{(-1)^{N_{2}}}{N_{2} !} \exp \left\{\beta\left(f_{2}-f_{2}^{\prime}\right) N_{2}\right\}\right]\left[\sum_{N_{3}=1}^{\infty} \frac{(-1)^{N_{3}}}{N_{3} !} \exp \left\{\beta\left(f_{3}-f_{3}^{\prime}\right) N_{3}\right\}\right]\right] \\
& =-\lim _{\beta \rightarrow \infty} \int_{-\infty}^{+\infty} d f_{1}^{\prime} \int_{-\infty}^{+\infty} d f_{2}^{\prime} \int_{-\infty}^{+\infty} d f_{3}^{\prime} P_{x, \epsilon, t}\left(f_{1}^{\prime}, f_{2}^{\prime}, f_{3}^{\prime}\right)\left[\exp \left\{-\exp \left[\beta\left(f_{1}-f_{1}^{\prime}\right)\right]\right\}-1\right] \times \\
& \times\left[\exp \left\{-\exp \left[\beta\left(f_{2}-f_{2}^{\prime}\right)\right]\right\}-1\right]\left[\exp \left\{-\exp \left[\beta\left(f_{3}-f_{3}^{\prime}\right)\right]\right\}-1\right] \\
& =\int_{-\infty}^{+\infty} d f_{1}^{\prime} \int_{-\infty}^{+\infty} d f_{2}^{\prime} \int_{-\infty}^{+\infty} d f_{3}^{\prime} P_{x, \epsilon, t}\left(f_{1}^{\prime}, f_{2}^{\prime}, f_{3}^{\prime}\right) \theta\left(f_{1}-f_{1}^{\prime}\right) \theta\left(f_{2}-f_{2}^{\prime}\right) \theta\left(f_{3}-f_{3}^{\prime}\right)
\end{aligned}
$$

which coincides with the definition (28).

Thus, according to eq.(29), in terms of the replica technique the probability function, eq.(28), can be represented as:

$$
W_{x, \epsilon, t}\left(f_{1}, f_{2}, f_{3}\right)=-\lim _{\beta \rightarrow \infty} \lim _{M \rightarrow 0} \sum_{N_{1}, N_{2}, N_{3}=1}^{\infty} \frac{(-1)^{N_{1}+N_{2}+N_{3}}}{N_{1} ! N_{2} ! N_{3} !} \exp \left\{\beta N_{1} f_{1}+\beta N_{2} f_{2}+\beta N_{3} f_{3}\right\} Z_{x, \epsilon, t}\left(M, N_{1}, N_{2}, N_{3}\right)
$$


where

$$
Z_{x, \epsilon, t}\left(M, N_{1}, N_{2}, N_{3}\right)=\overline{Z^{N_{1}}(x / 2, t) Z^{N_{2}}(x / 2-\epsilon, t) Z^{N_{3}}(-x / 2+\epsilon, t) Z^{M-N_{1}-N_{2}-N_{3}}(-x / 2, t)}
$$

Further program of calculations is in the following. The above four-point replica partition function has to be calculated for an integer $M>N_{1}+N_{2}+N_{3}$ as an analytic function of the parameter $M$. Then this function has to be analytically continued for arbitrary real values of $M$ and the limit $M \rightarrow 0$ has to be taken. Finally, after computing the series in eq.(31) (in the limits $t \rightarrow \infty$ and $\beta \rightarrow \infty$ ) according to the definition (28) the corresponding PDF $P_{x, \epsilon}\left(f_{1}, f_{2}, f_{3}\right)$ can be obtained as

$$
P_{x, \epsilon}\left(f_{1}, f_{2}, f_{3}\right)=\frac{\partial^{3}}{\partial f_{1} \partial f_{2} \partial f_{3}} \mathcal{W}_{x, \epsilon}\left(f_{1}, f_{2}, f_{3}\right)
$$

where

$$
\mathcal{W}_{x, \epsilon}\left(f_{1}, f_{2}, f_{3}\right) \equiv \lim _{\beta \rightarrow \infty} \lim _{t \rightarrow \infty} W_{x, \epsilon, t}\left(f_{1}, f_{2}, f_{3}\right)
$$

According to the representation (11) and the definitions (26) the velocities $v \equiv v(x / 2, t)$ and $v^{\prime} \equiv v(-x / 2, t)$ are defined as

$$
\begin{aligned}
v & =-\lim _{\epsilon \rightarrow 0} \frac{f_{1}-f_{2}}{\epsilon} \\
v^{\prime} & =-\lim _{\epsilon \rightarrow 0} \frac{f_{3}}{\epsilon}
\end{aligned}
$$

Thus, the corresponding joint PDF of these two velocities, $P_{x}\left(v, v^{\prime}\right)$ can be obtained as

$$
P_{x}\left(v, v^{\prime}\right)=\lim _{\epsilon \rightarrow 0}\left[\epsilon^{2} \int_{-\infty}^{+\infty} d f_{2} P_{x, \epsilon}\left(f_{2}-\epsilon v, f_{2},-\epsilon v^{\prime}\right)\right]
$$

The above general program of computations will be implemented in the further sections.

\section{ZERO TEMPERATURE LIMIT}

To compute the replica partition function, eq.(32), let us consider more general object:

$$
\Psi\left(x_{1}, x_{2}, \ldots, x_{M} ; t\right)=\overline{\left(\prod_{a=1}^{M} Z\left(x_{a}, t\right)\right)}
$$

Substituting here eqs.(77) and (44), and performing simple Gaussian averaging (using eq.(55)) we get

$$
\Psi\left(x_{1}, x_{2}, \ldots, x_{M} ; t\right)=\prod_{a=1}^{M}\left[\int_{\phi_{a}(0)=0}^{\phi_{a}(t)=x_{a}} \mathcal{D} \phi_{a}(\tau)\right] \exp \left\{-\beta H_{M}\left[\phi_{1}(\tau), \ldots, \phi_{M}(\tau)\right]\right\}
$$

where

$$
\beta H_{M}\left[\phi_{1}(\tau), \ldots, \phi_{M}(\tau)\right]=\int_{0}^{t} d \tau\left[\frac{1}{2} \beta \sum_{a=1}^{M}\left(\partial_{\tau} \phi_{a}(\tau)\right)^{2}-\frac{1}{2} \beta^{2} u \sum_{a, b=1}^{M} U\left(\phi_{a}(\tau)-\phi_{b}(\tau)\right)\right]
$$

is the replica Hamiltonian with the attractive interaction potential $U(\phi)$ given in eq.(6). One can easily show that the function $\Psi\left(x_{1}, x_{2}, \ldots, x_{M} ; t\right)$ is the wave function of one-dimensional quantum bosons which satisfy the imaginary time Schrödinger equation

$$
\beta \frac{\partial}{\partial t} \Psi(\mathbf{x} ; t)=\frac{1}{2} \sum_{a=1}^{M} \frac{\partial^{2}}{\partial x_{a}^{2}} \Psi(\mathbf{x} ; t)+\frac{1}{2} \beta^{3} u \sum_{a, b=1}^{M} U\left(x_{a}-x_{b}\right) \Psi(\mathbf{x} ; t)
$$

with the initial conditions $\Psi(\mathbf{x} ; 0)=\prod_{a=1}^{M} \delta\left(x_{a}\right)$ (here we have introduced the vector notation $\mathbf{x} \equiv\left\{x_{1}, x_{2}, \ldots, x_{M}\right\}$ ). 
The high temperature limit of the replica problem formulated above is well studied (for a review see e.g. 19] and references therein). It can be shown that in the limit $\beta \rightarrow 0$ the interaction potential $U(x)$, eq.(6), can be approximated by the $\delta$-function, and in this case the generic solution of the Schrödinger equation can be represented in terms of the Bethe ansatz eigenfunctions [20 22]. However, at low temperatures, $T \lesssim(u R)^{1 / 3}$, the typical distance between particles (defined by the wave function $\Psi(\mathbf{x})$ ) becomes comparable with the size $R$ of the interaction potential $U(x)$ and its approximation by the $\delta$-function is no longer valid. The zero temperature limit of the considered system has been studied in [17, 23]

In the limit of low temperatures it is convenient to redefine the parameters of the system in the following way:

$$
\begin{aligned}
\phi & =R \tilde{\phi} \\
\beta & =T_{*}^{-1} \tilde{\beta} \\
\tau & =\tau_{*} \tilde{\tau}
\end{aligned}
$$

where

$$
\begin{aligned}
T_{*} & =\left(\frac{u R}{\sqrt{2 \pi}}\right)^{1 / 3} \\
\tau_{*} & =\left(\sqrt{2 \pi} R^{5} u^{-1}\right)^{1 / 3}
\end{aligned}
$$

In the new notations the replica Hamiltonian (40) reads

$$
\beta H_{M}[\tilde{\boldsymbol{\phi}}]=\int_{0}^{t / \tau_{*}} d \tilde{\tau}\left[\frac{1}{2} \tilde{\beta} \sum_{a=1}^{M}\left(\partial_{\tilde{\tau}} \tilde{\phi}_{a}(\tilde{\tau})\right)^{2}-\frac{1}{2} \tilde{\beta}^{2} \sum_{a, b=1}^{M} U_{0}\left(\tilde{\phi}_{a}(\tilde{\tau})-\tilde{\phi}_{b}(\tilde{\tau})\right)\right]
$$

where

$$
U_{0}(\phi)=\exp \left\{-\frac{1}{2} \phi^{2}\right\}
$$

Accordingly, instead of eq.411) we get

$$
\tilde{\beta} \frac{\partial}{\partial \tilde{t}} \Psi(\tilde{\mathbf{x}} ; t)=\frac{1}{2} \sum_{a=1}^{M} \frac{\partial^{2}}{\partial \tilde{x}_{a}^{2}} \Psi(\tilde{\mathbf{x}} ; t)+\frac{1}{2} \tilde{\beta}^{3} \sum_{a, b=1}^{M} U_{0}\left(\tilde{x}_{a}-\tilde{x}_{b}\right) \Psi(\tilde{\mathbf{x}} ; t)
$$

where $\tilde{t}=t / \tau_{*}$ and $\tilde{x}=x / R$. Substituting here $\Psi(\tilde{\mathbf{x}} ; t)=\psi(\tilde{\mathbf{x}}) \exp \{-E \tilde{t}\}$ we obtain the following equation for the eigenfunctions $\psi(\tilde{\mathbf{x}})$ and the eigenvalues (energy) $E$ :

$$
-2 \tilde{\beta} E \psi(\tilde{\mathbf{x}})=\sum_{a=1}^{M} \frac{\partial^{2}}{\partial \tilde{x}_{a}^{2}} \psi(\tilde{\mathbf{x}})+\tilde{\beta}^{3} \sum_{a, b=1}^{M} U_{0}\left(\tilde{x}_{a}-\tilde{x}_{b}\right) \psi(\tilde{\mathbf{x}})
$$

which is controlled by the only parameter

$$
\tilde{\beta}=\beta T_{*}=\beta(u R)^{1 / 3}(2 \pi)^{-1 / 6}
$$

We see that $T_{*}$, eq.(43), is the crossover temperature which separates the high-temperatures, $T \gg T_{*}$, and the lowtemperatures, $T \ll T_{*}$, regimes. Note also that introduced above dimensionless inverse temperature parameter $\tilde{\beta}$, eq.(49), coincides with the Reynolds number Re, eq.(10), so that the limit of large Reynolds number in the Burgers problem corresponds to the zero temperature limit in the considered directed polymers model.

Recently it has been demonstrated [17] that in the limit $\tilde{\beta} \rightarrow \infty$ the eigenfunction $\psi(\tilde{\mathbf{x}})$ acquires specific vector replica symmetry breaking (RSB) coordinate structure, namely, $M$ its arguments $\left\{\tilde{x}_{1}, \ldots, \tilde{x}_{M}\right\}$ split into $K=M / m$ groups each consisting of $m$ particles. In other words, to describe the coordinate structure of the eigenfunction $\psi(\tilde{\mathbf{x}})$, instead of the particles coordinates $\left\{\tilde{x}_{a}\right\} \quad(a=1, \ldots, M)$ one introduces the coordinates of the center of masses of the groups $\left\{X_{\alpha}\right\} \quad(\alpha=1, \ldots, K)$ and the deviations $\left\{\xi_{i}^{\alpha}\right\} \quad(i=1, \ldots, m)$ of the particles of a given group $\alpha$ from the position of its center of mass:

$$
\tilde{x}_{a} \rightarrow X_{\alpha}+\xi_{i}^{\alpha} ; \quad \alpha=1, \ldots, M / m ; \quad i=1, \ldots, m
$$


where $\sum_{i=1}^{m} \xi_{i}^{\alpha}=0$. It can be shown [17] that in the zero temperature limit the typical value of the deviations insides groups are small, $\left.\left\langle\left(\xi_{i}^{\alpha}\right)^{2}\right\rangle\right|_{\tilde{\beta} \rightarrow \infty} \rightarrow 0$, while the typical distance between the groups remains finite. As these two spatial scales are well separated, the wave function $\psi(\tilde{\mathbf{x}})$ factorizes into the product of two contributions: the "external" wave function which depends only on the coordinates $\left\{X_{\alpha}\right\}$ of the center of masses of the groups, and the "internal" wave functions which depend only on the coordinates $\left\{\xi_{i}^{\alpha}\right\}$ of the particles inside the groups:

$$
\psi(\tilde{\mathbf{x}}) \rightarrow \psi\left(X_{\alpha} ; \xi_{i}^{\alpha}\right) \simeq \psi_{*}\left(X_{1}, \ldots, X_{M / m}\right) \times \prod_{\alpha=1}^{M / m} \psi_{0}\left(\xi_{1}^{\alpha}, \ldots \xi_{m}^{\alpha}\right)
$$

As the values $\xi_{i}^{\alpha}$ are small the interaction potential, eq.(46), between the particles inside groups can be approximated as

$$
U_{0}\left(\xi_{i}^{\alpha}-\xi_{j}^{\alpha}\right) \simeq 1-\frac{1}{2}\left(\xi_{i}^{\alpha}-\xi_{j}^{\alpha}\right)^{2}
$$

Thus, according to eq.(48), the corresponding equation for the "internal" eigenfunction $\psi_{0}(\boldsymbol{\xi})$ of any group reads

$$
-2 \tilde{\beta} E_{0} \psi_{0}(\boldsymbol{\xi})=\sum_{i=1}^{m} \frac{\partial^{2}}{\partial \xi_{i}^{2}} \psi_{0}(\boldsymbol{\xi})+\tilde{\beta}^{3} m^{2} \psi_{0}(\boldsymbol{\xi})-\frac{1}{2} \tilde{\beta}^{3} \sum_{i, j=1}^{m}\left(\xi_{i}-\xi_{j}\right)^{2} \psi_{0}(\boldsymbol{\xi})
$$

where $\boldsymbol{\xi}=\left\{\xi_{1}, \xi_{2}, \ldots, \xi_{m}\right\}$. One can easily show that this equation has the following exact (ground state) solution

$$
\psi_{0}(\boldsymbol{\xi})=C \exp \left\{-\frac{1}{4} \tilde{\beta}^{2}(\tilde{\beta} m)^{-1 / 2} \sum_{i, j=1}^{m}\left(\xi_{i}-\xi_{j}\right)^{2}\right\}
$$

where $C$ is the normalization constant and

$$
E_{0}=-\frac{1}{2}(\tilde{\beta} m)^{2}+\frac{1}{2}(m-1) \sqrt{\tilde{\beta} m}
$$

is the ground state energy.

On the other hand, the "external" wave function $\psi_{*}(\mathbf{X})$ (with $\mathbf{X}=\left\{X_{1}, \ldots, X_{M / m}\right\}$ ) is defined by the equation

$$
-2(\tilde{\beta} m) E_{*} \psi_{*}(\mathbf{X})=\sum_{\alpha=1}^{M / m} \frac{\partial^{2}}{\partial X_{\alpha}^{2}} \psi_{*}(\mathbf{X})+\frac{1}{2}(\tilde{\beta} m)^{3} \sum_{\alpha \neq \alpha^{\prime}}^{M / m} U_{0}\left(X_{\alpha}-X_{\alpha^{\prime}}\right) \psi_{*}(\mathbf{X})
$$

In terms of the replica approach, the parameter $m$ of the RSB ansatz described above is an integer such that $1 \leq m \leq M$ (so that $M / m$ is also an integer). In the framework of the standard replica technique, after computing the corresponding partition function and its analytic continuation for arbitrary (non-integer) values of $M$ and $m$, in the limit $M \rightarrow 0$ the parameter $m$ takes continuous (real) values at the interval $0 \leq m \leq 1$. Its actual physical value $m(\tilde{\beta})$ is fixed by the condition of the maximum of the total (linear in time $t \rightarrow \infty$ ) replica free energy. It can be shown [17] that in the limit $\tilde{\beta} \rightarrow \infty$ the value $m(\tilde{\beta})$ is defined by the relation

$$
\tilde{\beta} m=\zeta_{0}
$$

where $\zeta_{0}$ is a number of the order of one ( $\operatorname{such}$ that $m(\tilde{\beta}) \rightarrow 0$ as $\left.\tilde{\beta} \rightarrow \infty\right)$. The exact value of $\zeta_{0}$ is yet to be computed, as it is defined by the exact solution of the "external" problem, eq.(56), which at present is not known.

In terms of this RSB ansatz in the zero temperature limit the replica partition function of the considered system, eq.(32), factorizes into two parts:

$$
Z_{x, \epsilon, t}\left(M, N_{1}, N_{2}, N_{3}\right) \simeq Z_{*}[(\tilde{\beta} m), M / m, \tilde{t}] \times Z_{0}\left(M, m, \tilde{\beta}, N_{1}, N_{2}, N_{3}, x, \epsilon\right)
$$

where $Z_{*}$ is the "external" replica partition function:

$$
Z_{*}=\prod_{\alpha=1}^{M / m}\left[\int_{\varphi_{\alpha}(0)=0}^{\varphi_{\alpha}(\tilde{t})=0} \mathcal{D} \varphi_{\alpha}(\tau)\right] \exp \left\{-\frac{1}{2} \int_{0}^{\tilde{t}} d \tau\left[(\tilde{\beta} m) \sum_{\alpha=1}^{M / m}\left(\partial_{\tau} \varphi_{\alpha}\right)^{2}-(\tilde{\beta} m)^{2} \sum_{\alpha \neq \alpha^{\prime}}^{M / m} U_{0}\left(\varphi_{\alpha}-\varphi_{\alpha^{\prime}}\right)\right]-\tilde{t} \frac{M}{m} E_{0}\right\}
$$


where $E_{0}$ is given in eq.(155). Note that in the limit $\tilde{t} \rightarrow \infty$ this partition function is getting independent of $x$ and $\epsilon$, as these parameters are not scaling with $\tilde{t}$. The above "external" partition function $Z_{*}$ defines the extensive in $\tilde{t} \rightarrow \infty$ part of the directed polymer free energy and fixes the value of the parameter $m=m(\tilde{\beta})$, eq.(57), and it is in this way that the parameters of the large-scale random potential influence the small-scale statistics defined by the "internal" partition function (see below) which also depends on the value of $m(\tilde{\beta})$. On the other hand, by definition,

$$
\lim _{M \rightarrow 0} Z_{*}[(\tilde{\beta} m), M / m, \tilde{t}]=1
$$

and therefore, except for fixing the value of the replica parameter $m(\tilde{\beta})$, this part of the total partition function does not contribute to the probability function $\mathcal{W}_{x, \epsilon}\left(f_{1}, f_{2}, f_{3}\right)$, eqs.(34) and (31). This probability function is defined only by the "internal" (independent of $\tilde{t}$ ) partition function

$$
\begin{aligned}
\mathcal{Z}_{0}\left(N_{1}, N_{2}, N_{3} ; x, \epsilon\right) & =\lim _{\tilde{\beta} \rightarrow \infty} \lim _{M \rightarrow 0} Z_{0}\left(M, m, \tilde{\beta}, N_{1}, N_{2}, N_{3}, x, \epsilon\right) \\
& =\lim _{\tilde{\beta} \rightarrow \infty} \lim _{M \rightarrow 0}\left[\left.\sum_{\left\{\tilde{\xi}_{i}^{\alpha}\right\}} \prod_{\alpha=1}^{M / m} \psi_{0}\left(\tilde{\xi}_{1}^{\alpha}, \ldots, \tilde{\xi}_{m}^{\alpha}\right)\right|_{\left\{\tilde{\xi}_{i}^{\alpha}\right\}=(\tilde{x} / 2 ; \tilde{x} / 2-\epsilon ;-\tilde{x} / 2+\epsilon ;-\tilde{x} / 2)}\right]
\end{aligned}
$$

where the explicit expression for $\psi_{0}(\tilde{\boldsymbol{\xi}})$ is given in eq.(54), and where we have to sum over all possible distributions of $M$ particle coordinates $\left\{\tilde{\xi}_{i}^{\alpha}\right\} \quad(\alpha=1, \ldots, M / m ; i=1, \ldots, m)$ over four end-points $\tilde{x} / 2 ; \tilde{x} / 2-\epsilon ;-\tilde{x} / 2+\epsilon$ and $-\tilde{x} / 2$ with $\tilde{x}=x / R$ and $\tilde{\xi}_{i}^{\alpha}=\xi_{i}^{\alpha} / R$.

\section{FREE ENERGIES PROBABILITY DISTRIBUTION FUNCTION}

Substituting eqs.(54) and (49) as well as $\tilde{x}=x / R$ and $\tilde{\xi}_{i}^{\alpha}=\xi_{i}^{\alpha} / R$ into eq.(61) we gets

$$
\mathcal{Z}_{0}\left(N_{1}, N_{2}, N_{3} ; x, \epsilon\right)=\lim _{\beta \rightarrow \infty} \lim _{M \rightarrow 0}\left[\left.\sum_{\left\{\tilde{\xi}_{i}^{\alpha}\right\}} \prod_{\alpha=1}^{M / m} \exp \left\{-\frac{1}{4} \beta^{2} \gamma^{2} \sum_{i, j=1}^{m}\left(\xi_{i}^{\alpha}-\xi_{j}^{\alpha}\right)^{2}\right\}\right|_{\left\{\xi_{i}^{\alpha}\right\}=(x / 2 ; x / 2-\epsilon ;-x / 2+\epsilon ;-x / 2)}\right]
$$

where

$$
\gamma=\frac{T_{*}}{R(\tilde{\beta} m)^{1 / 4}}
$$

and $T_{*}$ is given in eq.(43). Note that the normalization factor $C$ of the wave function (54) can be dropped out in eq.(62), as $\lim _{M \rightarrow 0} C^{M / m}=1$. According to the definition, eq.(32), in the summation over various distributions of $M$ end-points $\xi_{i}^{\alpha}$ over four spatial points the total number of $\xi_{i}^{\alpha}$ 's attached to $x / 2, x / 2-\epsilon,-x / 2+\epsilon$ and $-x / 2$ are equal to $N_{1}, N_{2}, N_{3}$ and $\left(M-N_{1}-N_{2}-N_{3}\right)$ correspondingly. Let us denote the number of $\xi_{i}^{\alpha}$ 's of the group $\alpha$ attached to the points $x / 2, x / 2-\epsilon,-x / 2+\epsilon$ and $-x / 2$ by $k_{1}^{\alpha}, k_{2}^{\alpha}, k_{3}^{\alpha}$ and $k_{4}^{\alpha}$. As the total number of particles in each group is equal to $m$, by definition,

$$
k_{1}^{\alpha}+k_{2}^{\alpha}+k_{3}^{\alpha}+k_{4}^{\alpha}=m
$$

and

$$
\left\{\begin{array}{l}
\sum_{\alpha=1}^{M / m} k_{1}^{\alpha}=N_{1} \\
\sum_{\alpha=1}^{M / m} k_{2}^{\alpha}=N_{2} \\
\sum_{\alpha=1}^{M / m} k_{3}^{\alpha}=N_{3} \\
\sum_{\alpha=1}^{M / m} k_{4}^{\alpha}=M-N_{1}-N_{2}-N_{3}
\end{array}\right.
$$

Schematically the above replica structure of the partition function (62) is represented in Fig.2. Accordingly, the factors $\left(\xi_{i}^{\alpha}-\xi_{j}^{\alpha}\right)^{2}$ in eq. (62) can take four possible values: $\epsilon^{2},(x-\epsilon)^{2},(x-2 \epsilon)^{2}$ and $x^{2}$. Simple combinatoric 


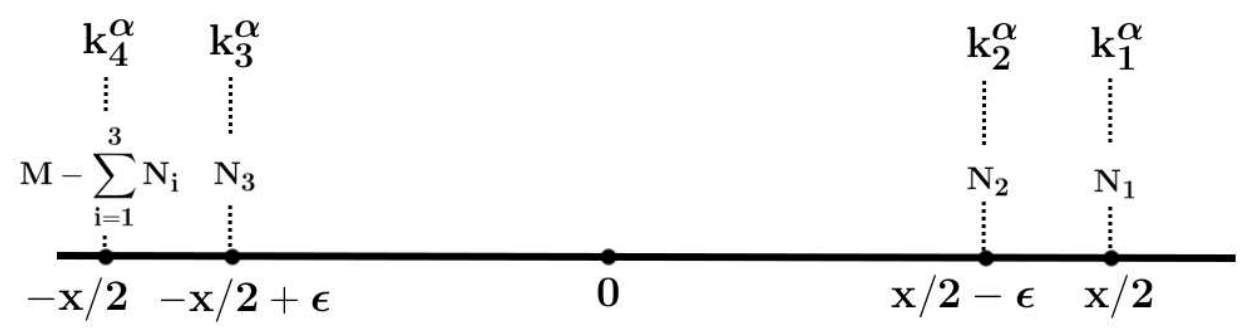

FIG. 2: Schematic representation of the replica structure of the partition function in eqs. (62)- (66).

considerations yield:

$$
\begin{aligned}
\mathcal{Z}_{0}\left(N_{1}, N_{2}, N_{3} ; x, \epsilon\right) & =\lim _{\beta \rightarrow \infty} \lim _{M \rightarrow 0}\left\{\frac{N_{1} ! N_{2} ! N_{3} !\left(M-N_{1}-N_{2}-N_{3}\right) !}{M !} \times\right. \\
& \times \prod_{\alpha=1}^{M / m}\left[\left(\prod_{i=1}^{4} \sum_{k_{i}^{\alpha}=0}^{m}\right) \frac{m !}{k_{1}^{\alpha} ! k_{2}^{\alpha} ! k_{3}^{\alpha} ! k_{4}^{\alpha} !} \delta\left(\sum_{i=1}^{4} k_{i}^{\alpha}, m\right) \exp \left\{-\frac{1}{4} \beta^{2} \gamma^{2} \sum_{i, j=1}^{4} D_{i j} k_{i}^{\alpha} k_{j}^{\alpha}\right\}\right] \times \\
& \left.\times \delta\left(\sum_{\alpha=1}^{M / m} k_{1}^{\alpha}, N_{1}\right) \delta\left(\sum_{\alpha=1}^{M / m} k_{2}^{\alpha}, N_{2}\right) \delta\left(\sum_{\alpha=1}^{M / m} k_{3}^{\alpha}, N_{3}\right)\right\}
\end{aligned}
$$

where $\boldsymbol{\delta}(p, q)$ is the Kronecker symbol and

$$
\hat{D}=\left(\begin{array}{cccc}
0 & \epsilon^{2} & (x-\epsilon)^{2} & x^{2} \\
\epsilon^{2} & 0 & (x-2 \epsilon)^{2} & (x-\epsilon)^{2} \\
(x-\epsilon)^{2} & (x-2 \epsilon)^{2} & 0 & \epsilon^{2} \\
x^{2} & (x-\epsilon)^{2} & \epsilon^{2} & 0
\end{array}\right)
$$

Note that the last constraint in eq.(65) can be dropped out of the expression (66), as it is automatically fulfilled due to the previous three ones together with the condition (64).

Substituting the matrix (67) into eq.(66) we get

$$
\begin{aligned}
\mathcal{Z}_{0}\left(N_{1}, N_{2}, N_{3} ; x, \epsilon\right) & =\lim _{\beta \rightarrow \infty} \lim _{M \rightarrow 0}\left\{\frac{N_{1} ! N_{2} ! N_{3} !\left(M-N_{1}-N_{2}-N_{3}\right) !}{M !} \times\right. \\
& \times \exp \left\{-\frac{1}{2} \beta N_{1}(\beta m) \gamma^{2} x^{2}-\frac{1}{2} \beta N_{2}(\beta m) \gamma^{2}(x-\epsilon)^{2}-\frac{1}{2} \beta N_{3}(\beta m) \gamma^{2} \epsilon^{2}\right\} \times \text { first } \\
& \times \prod_{\alpha=1}^{M / m}\left[\sum_{k_{1}^{\alpha}, k_{2}^{\alpha}, k_{3}^{\alpha}=0}^{m} C_{k_{1}^{\alpha}, k_{2}^{\alpha}, k_{3}^{\alpha}}^{m} \exp \left\{\frac{1}{2} \beta^{2} \gamma^{2}\left(x k_{1}^{\alpha}+(x-\epsilon) k_{2}^{\alpha}+\epsilon k_{3}^{\alpha}\right)^{2}\right\}\right] \times \\
& \left.\times \delta\left(\sum_{\alpha=1}^{M / m} k_{1}^{\alpha}, N_{1}\right) \delta\left(\sum_{\alpha=1}^{M / m} k_{2}^{\alpha}, N_{2}\right) \delta\left(\sum_{\alpha=1}^{M / m} k_{3}^{\alpha}, N_{3}\right)\right\}
\end{aligned}
$$

where

$$
C_{k_{1}^{\alpha}, k_{2}^{\alpha}, k_{3}^{\alpha}}^{m}=\frac{m !}{k_{1}^{\alpha} ! k_{2}^{\alpha} ! k_{3}^{\alpha} !\left(m-k_{1}^{\alpha}-k_{2}^{\alpha}-k_{3}^{\alpha}\right) !}
$$

Using the standard integral representation of the Kronecker symbol,

$$
\boldsymbol{\delta}(p, q)=\oint \frac{d z}{2 \pi i z} z^{p-q}
$$


(where contour of integration in the complex plane is the circle around zero) the partition function, eq.668), can be represented as follows:

$$
\begin{aligned}
\mathcal{Z}_{0}\left(N_{1}, N_{2}, N_{3} ; x, \epsilon\right) & =\lim _{\beta \rightarrow \infty} \lim _{M \rightarrow 0}\left\{\frac{N_{1} ! N_{2} ! N_{3} !\left(M-N_{1}-N_{2}-N_{3}\right) !}{M !} \exp \left\{-\beta N_{1} f_{01}-\beta N_{2} f_{02}-\beta N_{1} f_{03}\right\} \times\right. \\
& \times \frac{1}{(2 \pi i)^{3}} \oint \frac{d z_{1}}{z_{1}} z_{1}^{-N_{1}} \oint \frac{d z_{2}}{z_{2}} z_{2}^{-N_{2}} \oint \frac{d z_{3}}{z_{3}} z_{3}^{-N_{3}} \times \\
& \left.\times\left[\left\langle\left(1+z_{1} \exp \{\beta \gamma x \xi\}+z_{2} \exp \{\beta \gamma(x-\epsilon) \xi\}+z_{1} \exp \{\beta \gamma \epsilon \xi\}\right)^{m}\right]_{\xi}^{M / m}\right]^{M / m}\right\}
\end{aligned}
$$

where

$$
\begin{aligned}
f_{01} & =\frac{1}{2}(\beta m) \gamma^{2} x^{2} \\
f_{01} & =\frac{1}{2}(\beta m) \gamma^{2}(x-\epsilon)^{2} \\
f_{03} & =\frac{1}{2}(\beta m) \gamma^{2} \epsilon^{2}
\end{aligned}
$$

and $\langle(\ldots)\rangle_{\xi}$ denotes the Gaussian average over the variable $\xi$ :

$$
\langle(\ldots)\rangle_{\xi} \equiv \int_{-\infty}^{+\infty} \frac{d \xi}{\sqrt{2 \pi}}(\ldots) \exp \left\{-\frac{1}{2} \xi^{2}\right\}
$$

Now the expression for the replica partition function, eq.(71), can be analytically continued for arbitrary non-integer values of the parameter $M$. In particular, the factorial prefactor

$$
\frac{\left(M-N_{1}-N_{2}-N_{3}\right) !}{M !} \rightarrow \frac{\Gamma\left(M-N_{1}-N_{2}-N_{3}+1\right)}{\Gamma(M+1)}
$$

Using the Gamma function relation,

$$
\Gamma(-z)=-\frac{\pi}{\Gamma(z+1) \sin (\pi z)}
$$

for integer and positive values of $N_{1,2,3}$ this prefactor can be represented as follows,

$$
\begin{aligned}
\frac{\Gamma\left(M-N_{1}-N_{2}-N_{3}+1\right)}{\Gamma(M+1)} & =-\frac{\pi}{\Gamma(M+1) \Gamma\left(N_{1}+N_{2}+N_{3}-M\right) \sin \left[\pi\left(N_{1}+N_{2}+N_{3}-1\right)-\pi M\right]} \\
& =\frac{\pi(-1)^{N_{1}+N_{2}+N_{3}-1}}{\sin (\pi M) \Gamma\left(N_{1}+N_{2}+N_{3}-M\right) \Gamma(M+1)}
\end{aligned}
$$

so that in the limit $M \rightarrow 0$ we get

$$
\left.\frac{\pi(-1)^{N_{1}+N_{2}+N_{3}-1}}{\sin (\pi M) \Gamma\left(N_{1}+N_{2}+N_{3}-M\right) \Gamma(M+1)}\right|_{M \rightarrow 0} \rightarrow \frac{(-1)^{N_{1}+N_{2}+N_{3}-1}}{M \Gamma\left(N_{1}+N_{2}+N_{3}\right)}
$$

On the other hand, for the last factor in the expression (71) we find

$$
\left.[\ldots]^{M / m}\right|_{M \rightarrow 0} \rightarrow 1+\frac{M}{m} \ln [\ldots]
$$

Substituting eqs(77) and (78) into eq.(171) and taking into account that for any nonzero integer $N$,

$$
\oint \frac{d z_{1}}{z} z^{-N}=0
$$


in the limit $M \rightarrow 0$ we get

$$
\begin{aligned}
\mathcal{Z}_{0}\left(N_{1}, N_{2}, N_{3} ; x, \epsilon\right) & =\lim _{\beta \rightarrow \infty}\left\{\frac{(-1)^{N_{1}+N_{2}+N_{3}-1} \Gamma\left(N_{1}+1\right) \Gamma\left(N_{2}+1\right) \Gamma\left(N_{3}+1\right)}{m \Gamma\left(N_{1}+N_{2}+N_{3}\right)} \exp \left\{-\beta N_{1} f_{01}-\beta N_{2} f_{02}-\beta N_{1} f_{03}\right\} \times\right. \\
& \times \frac{1}{(2 \pi i)^{3}} \oint \frac{d z_{1}}{z_{1}} z_{1}^{-N_{1}} \oint \frac{d z_{2}}{z_{2}} z_{2}^{-N_{2}} \oint \frac{d z_{3}}{z_{3}} z_{3}^{-N_{3}} \times \\
& \left.\times \ln \left[\left\langle\left(1+z_{1} \exp \{\beta \gamma x \xi\}+z_{2} \exp \{\beta \gamma(x-\epsilon) \xi\}+z_{1} \exp \{\beta \gamma \epsilon \xi\}\right)^{m}\right\rangle_{\xi}\right]\right\}
\end{aligned}
$$

Substituting this expression into eqs.(31) and (34) for the free energy probability distribution function we obtain

$$
\begin{aligned}
\mathcal{W}_{x, \epsilon}\left(f_{1}, f_{2}, f_{3}\right) & =\lim _{\beta \rightarrow \infty}\left\{\sum_{N_{1}, N_{2}, N_{3}=1}^{\infty} \frac{\exp \left\{\beta N_{1}\left(f_{1}-f_{01}\right)+\beta N_{2}\left(f_{1}-f_{02}\right)+\beta N_{1}\left(f_{1}-f_{03}\right)\right\}}{m \Gamma\left(N_{1}+N_{2}+N_{3}\right)} \times\right. \\
& \times \frac{1}{(2 \pi i)^{3}} \oint \frac{d z_{1}}{z_{1}} z_{1}^{-N_{1}} \oint \frac{d z_{2}}{z_{2}} z_{2}^{-N_{2}} \oint \frac{d z_{3}}{z_{3}} z_{3}^{-N_{3}} \times \\
& \left.\times \ln \left[\left\langle\left(1+z_{1} \exp \{\beta \gamma x \xi\}+z_{2} \exp \{\beta \gamma(x-\epsilon) \xi\}+z_{1} \exp \{\beta \gamma \epsilon \xi\}\right)^{m}\right\rangle_{\xi}\right]\right\}
\end{aligned}
$$

The limit $\beta \rightarrow \infty$ is somewhat tricky: on one hand, according to eq. (57) in the zero temperature limit $m \propto 1 / \beta \rightarrow 0$ and on the other hand we have several exponential factors in the above expression which are formally divergent in this limit. To take the limit $m \propto 1 / \beta \rightarrow 0$ the expression under the logarithm in eq.(81) can be represented as follows:

$$
\begin{aligned}
& \left\langle\left(1+z_{1} \exp \{\beta \gamma x \xi\}+z_{2} \exp \{\beta \gamma(x-\epsilon) \xi\}+z_{1} \exp \{\beta \gamma \epsilon \xi\}\right)^{m}\right\rangle_{\xi}= \\
= & 1+\sum_{k_{1}+k_{2}+k_{3} \geq 1}^{\infty} C_{k_{1} k_{2} k_{3}}^{m} z_{1}^{k_{1}} z_{2}^{k_{2}} z_{3}^{k_{3}}\left\langle\exp \left\{\beta k_{1} \gamma x \xi+\beta k_{2} \gamma(x-\epsilon) \xi+\beta k_{3} \gamma \epsilon \xi\right\}\right\rangle_{\xi}
\end{aligned}
$$

where

$$
C_{k_{1} k_{2} k_{3}}^{m}=\frac{\Gamma(m+1)}{\Gamma\left(k_{1}+1\right) \Gamma\left(k_{2}+1\right) \Gamma\left(k_{3}+1\right) \Gamma\left(m-k_{1}-k_{2}-k_{3}+1\right)}
$$

In the limit $m \rightarrow 0$ we get (sf eqs.(76)-(77))

$$
\left.C_{k_{1} k_{2} k_{3}}^{m}\right|_{m \rightarrow 0} \simeq m \frac{(-1)^{k_{1}+k_{2}+k_{3}-1}}{k_{1}+k_{2}+k_{3}} C_{k_{1} k_{2} k_{3}}^{0}
$$

where

$$
C_{k_{1} k_{2} k_{3}}^{0}=\frac{\Gamma\left(k_{1}+k_{2}+k_{3}+1\right)}{\Gamma\left(k_{1}+1\right) \Gamma\left(k_{2}+1\right) \Gamma\left(k_{3}+1\right)}
$$

Substituting eqs.(84) and (82) into eq.(81) and expending the logarithm term after integrations over $z_{1}, z_{2}$ and $z_{3}$ we obtain

$$
\begin{aligned}
& \mathcal{W}_{x, \epsilon}\left(f_{1}, f_{2}, f_{3}\right)=\lim _{\beta \rightarrow \infty}\left\{\frac{1}{(\beta m)} \sum_{n=1}^{\infty} \frac{(-1)^{n-1}}{n} \times\right. \\
& \times \prod_{\alpha=1}^{n}\left[(\beta m) \sum_{k_{1}^{\alpha}+k_{2}^{\alpha}+k_{3}^{\alpha} \geq 1}^{\infty} \frac{(-1)^{k_{1}^{\alpha}+k_{2}^{\alpha}+k_{3}^{\alpha}-1}}{\beta\left(k_{1}^{\alpha}+k_{2}^{\alpha}+k_{3}^{\alpha}\right)} C_{k_{1}^{\alpha} k_{2}^{\alpha} k_{3}^{\alpha}}^{0}\left\langle\exp \left\{\beta k_{1}^{\alpha} \gamma x \xi+\beta k_{2}^{\alpha} \gamma(x-\epsilon) \xi+\beta k_{3}^{\alpha} \gamma \epsilon \xi\right\}\right\rangle_{\xi}\right] \times \\
& \times \sum_{N_{1}, N_{2}, N_{3}=1}^{\infty} \frac{\beta\left(N_{1}+N_{2}+N_{3}\right)}{\Gamma\left(N_{1}+N_{2}+N_{3}+1\right)} \exp \left\{\beta N_{1}\left(f_{1}-f_{01}\right)+\beta N_{2}\left(f_{1}-f_{02}\right)+\beta N_{1}\left(f_{1}-f_{03}\right)\right\} \times \\
& \left.\times \delta\left(\sum_{\alpha=1}^{n} k_{1}^{\alpha}, N_{1}\right) \delta\left(\sum_{\alpha=1}^{n} k_{2}^{\alpha}, N_{2}\right) \boldsymbol{\delta}\left(\sum_{\alpha=1}^{n} k_{3}^{\alpha}, N_{3}\right)\right\}
\end{aligned}
$$


Substituting here $\beta m=\tilde{\beta} m / T_{*}=\zeta_{0} / T_{*}$ (see eqs.(57), (42) and (43)) and resolving the Kronecker symbols in the summations over $N_{1}, N_{2}$ and $N_{3}$ we get

$$
\begin{aligned}
& \mathcal{W}_{x, \epsilon}\left(f_{1}, f_{2}, f_{3}\right)=\frac{T_{*}}{\zeta_{0}} \lim _{\beta \rightarrow \infty}\left\{\sum _ { n = 1 } ^ { \infty } \frac { ( - 1 ) ^ { n - 1 } } { n } ( \frac { \zeta _ { 0 } } { T _ { * } } ) ^ { n } \prod _ { \alpha = 1 } ^ { n } \left[\sum_{k_{1}^{\alpha}+k_{2}^{\alpha}+k_{3}^{\alpha} \geq 1}^{\infty} \frac{(-1)^{k_{1}^{\alpha}+k_{2}^{\alpha}+k_{3}^{\alpha}-1}}{\beta\left(k_{1}^{\alpha}+k_{2}^{\alpha}+k_{3}^{\alpha}\right)} C_{k_{1}^{\alpha} k_{2}^{\alpha} k_{3}^{\alpha} \times} \times\right.\right. \\
\times & \left.\left\langle\exp \left\{\beta k_{1}^{\alpha}\left(\gamma x \xi+f_{1}-f_{01}\right)+\beta k_{2}^{\alpha}\left(\gamma(x-\epsilon) \xi+f_{2}-f_{02}\right)+\beta k_{3}^{\alpha}\left(\gamma \epsilon \xi+f_{3}-f_{03}\right)\right\}\right\rangle_{\xi}\right] \times \\
\times & \left.\frac{\beta \sum_{\alpha=1}^{n}\left(k_{1}^{\alpha}+k_{2}^{\alpha}+k_{3}^{\alpha}\right)}{\Gamma\left[\sum_{\alpha=1}^{n}\left(k_{1}^{\alpha}+k_{2}^{\alpha}+k_{3}^{\alpha}\right)+1\right]} \boldsymbol{\theta}\left(\sum_{\alpha=1}^{n} k_{1}^{\alpha}-1\right) \boldsymbol{\theta}\left(\sum_{\alpha=1}^{n} k_{2}^{\alpha}-1\right) \boldsymbol{\theta}\left(\sum_{\alpha=1}^{n} k_{3}^{\alpha}-1\right)\right\}
\end{aligned}
$$

where the symbol $\boldsymbol{\theta}(p-1)$ (the "discrete step function") indicates that $p \geq 1$. Note however, that according to eq. (87) the contributions with $\sum_{\alpha=1}^{n} k_{i}^{\alpha}=0$ (such that all $k_{i}^{1}=k_{1}^{2}=\ldots=k_{i}^{n}=0$ ) are independent of the corresponding free energy parameter $f_{i}$. On the other hand, the probability density function $P_{x, \epsilon}\left(f_{1}, f_{2}, f_{3}\right)$ which we are aiming to derive is given by the derivatives of the above probability function $W$ over all three variables $f_{1}, f_{2}$ and $f_{3}$ (see eq.(33) ). Therefore, as far as the $\operatorname{PDF} P_{x, \epsilon}\left(f_{1}, f_{2}, f_{3}\right)$ is concerned the restrictions imposed by the last three "discrete step function" in eq.(87) can be omitted.

The factor $\beta \sum_{\alpha=1}^{n}\left(k_{1}^{\alpha}+k_{2}^{\alpha}+k_{3}^{\alpha}\right)$ in the numerator of the last term in eq.(87) can be obtained by taking the derivatives $\left(\frac{\partial}{\partial f_{1}}+\frac{\partial}{\partial f_{2}}+\frac{\partial}{\partial f_{3}}\right)$ of $\mathcal{W}_{x, \epsilon}\left(f_{1}, f_{2}, f_{3}\right)$. On the other hand,

$$
\frac{1}{\beta\left(k_{1}^{\alpha}+k_{2}^{\alpha}+k_{3}^{\alpha}\right)}=\int_{0}^{+\infty} d y \exp \left\{-\beta\left(k_{1}^{\alpha}+k_{2}^{\alpha}+k_{3}^{\alpha}\right) y\right\}
$$

Substituting this into eq.(87) and then, substituting the obtained expression into eq.(33) for the PDF $P_{x, \epsilon}\left(f_{1}, f_{2}, f_{3}\right)$ we get

$$
\begin{aligned}
& P_{x, \epsilon}\left(f_{1}, f_{2}, f_{3}\right)=\frac{T_{*}}{\zeta_{0}} \frac{\partial^{3}}{\partial f_{1} \partial f_{2} \partial f_{3}}\left(\frac{\partial}{\partial f_{1}}+\frac{\partial}{\partial f_{2}}+\frac{\partial}{\partial f_{3}}\right) \lim _{\beta \rightarrow \infty}\left\{\sum_{n=1}^{\infty} \frac{(-1)^{n-1}}{n}\left(\frac{\zeta_{0}}{T_{*}}\right)^{n} \times\right. \\
\times & \prod_{\alpha=1}^{n}\left[\int_{0}^{+\infty} d y \sum_{k_{1}^{\alpha}+k_{2}^{\alpha}+k_{3}^{\alpha} \geq 1}^{\infty}(-1)^{k_{1}^{\alpha}+k_{2}^{\alpha}+k_{3}^{\alpha}-1} C_{k_{1}^{\alpha} k_{2}^{\alpha} k_{3}^{\alpha} \times} \times\right. \\
\times & \left.\left\langle\exp \left\{\beta k_{1}^{\alpha}\left(\gamma x \xi+f_{1}-f_{01}-y\right)+\beta k_{2}^{\alpha}\left(\gamma(x-\epsilon) \xi+f_{2}-f_{02}-y\right)+\beta k_{3}^{\alpha}\left(\gamma \epsilon \xi+f_{3}-f_{03}-y\right)\right\}\right\rangle_{\xi}\right] \times \\
\times & \left.\frac{1}{\Gamma\left[\sum_{\alpha=1}^{n}\left(k_{1}^{\alpha}+k_{2}^{\alpha}+k_{3}^{\alpha}\right)+1\right]}\right\}
\end{aligned}
$$

In the limit $\beta \rightarrow \infty$ the summation the series over $k_{i}^{\alpha}$ in the above expression can be done using the their integral representation. Namely, let us consider the series of a general type

$$
R(\beta)=\sum_{k=0}^{\infty}(-1)^{k-1} \Phi(\beta k ; k)
$$

where $\Phi\left(z, z^{\prime}\right)$ is a "good" analytic function in the complex plane. One can easily see that the summation in eq. (90) can be changed by the integration in the complex plane:

$$
R(\beta)=\frac{1}{2 i} \int_{\mathcal{C}} \frac{d z}{\sin (\pi z)} \Phi(\beta z ; z)
$$

where the integration goes over the contour $\mathcal{C}$ shown in Fig.3, and it is assumed that the function $\Phi$ is such that its integration at infinity gives no contribution. Indeed, due to the sign alternating contributions of simple poles at integer $z=1,2, \ldots$ eq.(91) reduces to eq.(90). Then, redefining $z \rightarrow z / \beta$ we gets

$$
\lim _{\beta \rightarrow \infty} R(\beta)=\frac{1}{2 \pi i} \int_{\mathcal{C}} \frac{d z}{z} \lim _{\beta \rightarrow \infty} \Phi(z ; z / \beta)
$$




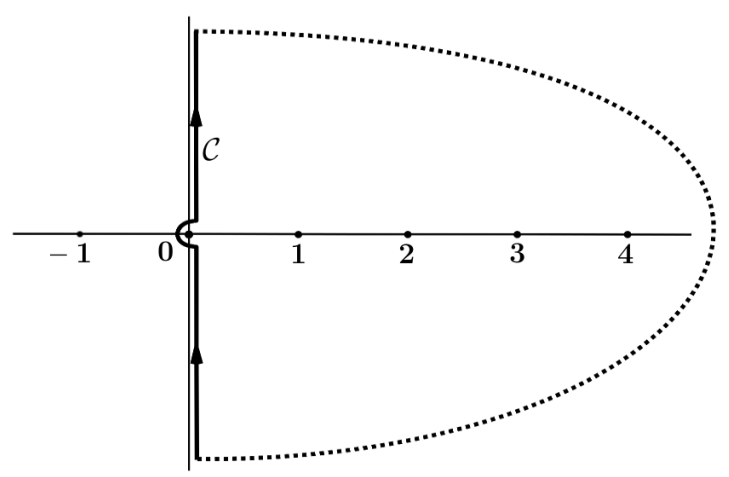

FIG. 3: The contour of integration in eq.910

In terms of the above integral representation, changing $k_{i}^{\alpha} \rightarrow z_{i}^{\alpha} / \beta$ for the Gamma function factors in eq.(89) we have:

$$
\left.\Gamma\left[\sum_{\alpha=1}^{n}\left(k_{1}^{\alpha}+k_{2}^{\alpha}+k_{3}^{\alpha}\right)+1\right] \rightarrow \Gamma\left[\sum_{\alpha=1}^{n}\left(z_{1}^{\alpha}+z_{2}^{\alpha}+z_{3}^{\alpha}\right) / \beta+1\right]\right|_{\beta \rightarrow \infty} \rightarrow 1
$$

and (see eq.

$$
\left.C_{k_{1}^{\alpha} k_{2}^{\alpha} k_{3}^{\alpha}}^{0} \rightarrow \frac{\Gamma\left(z_{1}^{\alpha} / \beta+z_{2}^{\alpha} / \beta+z_{3}^{\alpha} / \beta+1\right)}{\Gamma\left(z_{1}^{\alpha} / \beta+1\right) \Gamma\left(z_{2}^{\alpha} / \beta+1\right) \Gamma\left(z_{3}^{\alpha} / \beta+1\right)}\right|_{\beta \rightarrow \infty} \rightarrow 1
$$

Thus, after extracting the contributions with $k_{1}^{\alpha}=k_{2}^{\alpha}=k_{3}^{\alpha}=0$, the expression in eq.(89) reduces to

$$
\begin{aligned}
& P_{x, \epsilon}\left(f_{1}, f_{2}, f_{3}\right)=\frac{T_{*}}{\zeta_{0}} \frac{\partial^{3}}{\partial f_{1} \partial f_{2} \partial f_{3}}\left(\frac{\partial}{\partial f_{1}}+\frac{\partial}{\partial f_{2}}+\frac{\partial}{\partial f_{3}}\right) \sum_{n=1}^{\infty} \frac{(-1)^{n-1}}{n}\left(\frac{\zeta_{0}}{T_{*}}\right)^{n} \times \\
\times & {\left[\int _ { 0 } ^ { + \infty } d y \left\langle\left(\int_{\mathcal{C}} \frac{d z_{1}}{2 \pi i z_{1}} \exp \left\{z_{1}\left(\gamma x \xi+f_{1}-f_{01}-y\right)\right\} \int_{\mathcal{C}} \frac{d z_{2}}{2 \pi i z_{2}} \exp \left\{z_{2}\left(\gamma(x-\epsilon) \xi+f_{2}-f_{02}-y\right)\right\} \times\right.\right.\right.} \\
\times & \left.\left.\left.\int_{\mathcal{C}} \frac{d z_{3}}{2 \pi i z_{3}} \exp \left\{z_{3}\left(\gamma \epsilon \xi+f_{3}-f_{03}-y\right)\right\}+1\right)\right\rangle_{\xi}\right]^{n}
\end{aligned}
$$

Taking into account that

$$
\int_{\mathcal{C}} \frac{d z}{2 \pi i z} \exp \{\lambda z\}=-\theta(-\lambda)
$$

we obtain

$$
P_{x, \epsilon}\left(f_{1}, f_{2}, f_{3}\right)=\frac{T_{*}}{\zeta_{0}} \frac{\partial^{3}}{\partial f_{1} \partial f_{2} \partial f_{3}}\left(\frac{\partial}{\partial f_{1}}+\frac{\partial}{\partial f_{2}}+\frac{\partial}{\partial f_{3}}\right) \ln \left[1+S\left(f_{1}, f_{2}, f_{3}\right)\right]
$$

or

$$
P_{x, \epsilon}\left(f_{1}, f_{2}, f_{3}\right)=\frac{\partial^{3}}{\partial f_{1} \partial f_{2} \partial f_{3}}\left[\left(1+S\left(f_{1}, f_{2}, f_{3}\right)\right)^{-1} G\left(f_{1}, f_{2}, f_{3}\right)\right]
$$

where

$$
\begin{aligned}
S\left(f_{1}, f_{2}, f_{3}\right) & =\frac{\zeta_{0}}{T_{*}} \int_{0}^{+\infty} d y \int_{-\infty}^{+\infty} \frac{d \xi}{\sqrt{2 \pi}} \exp \left\{-\frac{1}{2} \xi^{2}\right\} \times \\
& \times\left[1-\theta\left(y+f_{01}-f_{1}-\gamma x \xi\right) \theta\left(y+f_{02}-f_{2}-\gamma(x-\epsilon) \xi\right) \theta\left(y+f_{03}-f_{3}-\gamma \epsilon \xi\right)\right]
\end{aligned}
$$


and

$$
\begin{aligned}
G\left(f_{1}, f_{2}, f_{3}\right) & =\frac{T_{*}}{\zeta_{0}}\left(\frac{\partial}{\partial f_{1}}+\frac{\partial}{\partial f_{2}}+\frac{\partial}{\partial f_{3}}\right) S\left(f_{1}, f_{2}, f_{3}\right) \\
& =\int_{-\infty}^{+\infty} \frac{d \xi}{\sqrt{2 \pi}} \exp \left\{-\frac{1}{2} \xi^{2}\right\}\left[1-\theta\left(f_{01}-f_{1}-\gamma x \xi\right) \theta\left(f_{02}-f_{2}-\gamma(x-\epsilon) \xi\right) \theta\left(f_{03}-f_{3}-\gamma \epsilon \xi\right)\right]
\end{aligned}
$$

\section{TWO VELOCITY PROBABILITY DENSITY FUNCTION}

In this Section using the general result for the directed polymers three-point free energy distribution function, eqs. (98)-(100), we are going to derive two velocity probability density function $P_{x}\left(v, v^{\prime}\right)$ of the corresponding randomly forced Burgers problem. According to the discussion of Section II, eqs.(35)-(37),

$$
P_{x}\left(v, v^{\prime}\right)=\lim _{\epsilon \rightarrow 0}\left[\epsilon^{2} \int_{-\infty}^{+\infty} d f_{2} P_{x, \epsilon}\left(f_{2}-\epsilon v, f_{2},-\epsilon v^{\prime}\right)\right]
$$

where $v$ and $v^{\prime}$ are two velocities at two spatial points separated by the distance $x$. Explicitly, the expression for the function $P_{x, \epsilon}\left(f_{1}, f_{2}, f_{3}\right)$, eq.(98), reads

$$
\begin{aligned}
P_{x, \epsilon}\left(f_{1}, f_{2}, f_{3}\right) & =(1+S)^{-1} G_{123}^{\prime \prime \prime}- \\
& -(1+S)^{-2}\left[S_{1}^{\prime} G_{23}^{\prime \prime}+S_{2}^{\prime} G_{13}^{\prime \prime}+S_{3}^{\prime} G_{12}^{\prime \prime}+S_{12}^{\prime \prime} G_{3}^{\prime}+S_{13}^{\prime \prime} G_{2}^{\prime}+S_{23}^{\prime \prime} G_{1}^{\prime}+S_{123}^{\prime \prime \prime} G\right]+ \\
& +2(1+S)^{-3}\left[S_{1}^{\prime} S_{2}^{\prime} G_{3}^{\prime}+S_{1}^{\prime} S_{3}^{\prime} G_{2}^{\prime}+S_{2}^{\prime} S_{3}^{\prime} G_{1}^{\prime}+\left(S_{1}^{\prime} S_{23}^{\prime \prime}+S_{2}^{\prime} S_{13}^{\prime \prime}+S_{3}^{\prime} S_{12}^{\prime \prime}\right) G\right]- \\
& -6(1+S)^{-4} S_{1}^{\prime} S_{2}^{\prime} S_{3}^{\prime} G
\end{aligned}
$$

where we have introduced the notations $\Phi_{i}^{\prime} \equiv \frac{\partial}{\partial f_{i}} \Phi$ and the functions $S=S\left(f_{1}, f_{2}, f_{3}\right)$ and $G=G\left(f_{1}, f_{2}, f_{3}\right)$ are given in eqs.(99) and (100). Substituting this expression into eq.(101) we find that the only non-zero contributions in the limit $\epsilon \rightarrow 0$ come from two terms in the r.h.s. of eq.(102): $(1+S)^{-1} G_{123}^{\prime \prime \prime}$ and $-(1+S)^{-2} S_{12}^{\prime \prime} G_{3}^{\prime}$ (both of which $\propto 1 / \epsilon^{2}$ ) where (see Appendix A),

$$
\begin{aligned}
\left.G_{123}^{\prime \prime \prime}\right|_{\epsilon \rightarrow 0} & =\frac{x}{\epsilon^{2} \gamma \sqrt{2 \pi}} \exp \left\{-\frac{1}{2 \gamma^{2}}\left(v^{\prime}\right)^{2}\right\} \delta\left(f_{2}-f_{0}+x v^{\prime}\right) \delta\left(x v^{\prime}-x v-2 f_{0}\right) \\
\left.G_{3}^{\prime}\right|_{\epsilon \rightarrow 0} & =\frac{1}{\epsilon \gamma \sqrt{2 \pi}} \exp \left\{-\frac{1}{2 \gamma^{2}}\left(v^{\prime}\right)^{2}\right\} \theta\left(f_{0}-f_{2}-x v^{\prime}\right) \\
\left.S_{12}^{\prime \prime}\right|_{\epsilon \rightarrow 0} & =-\frac{\zeta_{0}}{\epsilon \gamma T_{*} \sqrt{2 \pi}} \exp \left\{-\frac{1}{2 \gamma^{2} x^{2}}\left(x v+2 f_{0}\right)^{2}\right\} \theta\left(x v+f_{0}+f_{2}\right)
\end{aligned}
$$

Here, according to eqs.(172), (63), (157) and (49),

$$
\begin{gathered}
f_{0} \equiv f_{01}=\frac{1}{2}(\beta m) \gamma^{2} x^{2}=\frac{1}{2} \sqrt{\zeta_{0}} T_{*} \cdot \frac{x^{2}}{R^{2}} \\
\gamma=\zeta_{0}^{-1 / 4} \frac{T_{*}}{R}
\end{gathered}
$$

and $T_{*}$ given in eq.(43). Using explicit expression for $S\left(f_{1}, f_{2}, f_{3}\right)$, eq.(99), one finds

$$
\lim _{\epsilon \rightarrow 0} S\left(f_{2}-\epsilon v, f_{2},-\epsilon v^{\prime}\right)=\frac{\zeta_{0}}{\gamma T_{*} x} \int_{0}^{\infty} \frac{d \xi}{\sqrt{2 \pi}} \xi \exp \left\{-\frac{1}{2 \gamma^{2} x^{2}}\left(\xi+f_{0}-f_{2}\right)^{2}\right\}
$$


Substituting eqs.(102)-(108) into eq.(101) we get

$$
\begin{aligned}
P_{x}\left(v, v^{\prime}\right) & =\int_{-\infty}^{+\infty} d f_{2}\left\{\frac{x}{\gamma \sqrt{2 \pi}} \frac{\exp \left\{-\frac{1}{2 \gamma^{2}}\left(v^{\prime}\right)^{2}\right\} \delta\left(f_{2}-f_{0}+x v^{\prime}\right) \delta\left(x v^{\prime}-x v-2 f_{0}\right)}{1+\frac{\zeta_{0}}{\gamma T_{*} x} \int_{0}^{\infty} \frac{d \xi}{\sqrt{2 \pi}} \xi \exp \left\{-\frac{1}{2 \gamma^{2} x^{2}}\left(\xi+f_{0}-f_{2}\right)^{2}\right\}}+\right. \\
& \left.+\frac{\zeta_{0}}{2 \pi \gamma^{2} T_{*}} \frac{\exp \left\{-\frac{1}{2 \gamma^{2} x^{2}}\left(x v+2 f_{0}\right)^{2}-\frac{1}{2 \gamma^{2}}\left(v^{\prime}\right)^{2}\right\} \theta\left(x v+f_{0}+f_{2}\right) \theta\left(f_{0}-f_{2}-x v^{\prime}\right)}{\left[1+\frac{\zeta_{0}}{\gamma T_{*} x} \int_{0}^{\infty} \frac{d \xi}{\sqrt{2 \pi}} \xi \exp \left\{-\frac{1}{2 \gamma^{2} x^{2}}\left(\xi+f_{0}-f_{2}\right)^{2}\right\}\right]^{2}}\right\}
\end{aligned}
$$

Introducing the notation (sf eq.(9) )

$$
v_{0}=\zeta_{0}^{3 / 4} \gamma=\sqrt{\zeta_{0}} \frac{T_{*}}{R}=\left(\frac{\zeta_{0}^{3}}{2 \pi}\right)^{1 / 6}\left(\frac{u}{R^{2}}\right)^{1 / 3}
$$

and changing the integration variables, $f_{2} \rightarrow f_{0}-x v_{0} \eta, \xi \rightarrow \gamma x \xi$ we eventually get the following result for the joint probability density function of two velocities at the distance $x$ :

$$
P_{x}\left(v, v^{\prime}\right)=p_{0}(v, x) \delta\left(v^{\prime}-v-v_{0} \frac{x}{R}\right)+\mathcal{P}_{x}\left(v, v^{\prime}\right) \theta\left(v+v_{0} \frac{x}{R}-v^{\prime}\right)
$$

where

$$
p_{0}(v, x)=\frac{\zeta_{0}^{3 / 4}}{v_{0} \sqrt{2 \pi}} \frac{\exp \left\{-\frac{1}{2} \zeta_{0}^{3 / 2}\left(\frac{v}{v_{0}}+\frac{x}{R}\right)^{2}\right\}}{\left[1+\zeta_{0}^{3 / 4} \frac{x}{R} \int_{0}^{\infty} \frac{d \xi}{\sqrt{2 \pi}} \xi \exp \left\{-\frac{1}{2}\left[\xi+\zeta_{0}^{3 / 4}\left(\frac{v}{v_{0}}+\frac{x}{R}\right)\right]^{2}\right\}\right]}
$$

and

$$
\mathcal{P}_{x}\left(v, v^{\prime}\right)=\frac{\zeta_{0}^{3} x}{2 \pi v_{0}^{2} R} \int_{v^{\prime} / v_{0}}^{v / v_{0}+x / R} d \eta \frac{\exp \left\{-\frac{1}{2} \zeta_{0}^{3 / 2}\left[\left(\frac{v}{v_{0}}+\frac{x}{R}\right)^{2}+\left(\frac{v^{\prime}}{v_{0}}\right)^{2}\right]\right\}}{\left[1+\zeta_{0}^{3 / 4} \frac{x}{R} \int_{0}^{\infty} \frac{d \xi}{\sqrt{2 \pi}} \xi \exp \left\{-\frac{1}{2}\left(\xi+\zeta_{0}^{3 / 4} \eta\right)^{2}\right\}\right]^{2}}
$$

\section{PROBABILITY DISTRIBUTION FUNCTION OF THE VELOCITY DIFFERENCE}

Using the joint distribution function $P_{x}\left(v, v^{\prime}\right)$ of two velocities $v$ and $v^{\prime}$ at distance $x$ derived above, eqs.(111)-(113), the probability density function of the velocity difference $w=v^{\prime}-v$ can be obtained as follows

$$
P_{x}(w)=\int_{-\infty}^{+\infty} d v P_{x}(v, v+w)
$$

Substituting here eqs.(111)-(113) we get

$$
P_{x}(w)=p_{0}(x) \delta\left(w-v_{0} \frac{x}{R}\right)+\mathcal{P}_{x}(w) \theta\left(v_{0} \frac{x}{R}-w\right)
$$

where

$$
p_{0}(x)=\frac{\zeta_{0}^{3 / 4}}{v_{0} \sqrt{2 \pi}} \int_{-\infty}^{+\infty} d v \frac{\exp \left\{-\frac{1}{2} \zeta_{0}^{3 / 2}\left(\frac{v}{v_{0}}+\frac{x}{R}\right)^{2}\right\}}{\left[1+\zeta_{0}^{3 / 4} \frac{x}{R} \int_{0}^{\infty} \frac{d \xi}{\sqrt{2 \pi}} \xi \exp \left\{-\frac{1}{2}\left[\xi+\zeta_{0}^{3 / 4}\left(\frac{v}{v_{0}}+\frac{x}{R}\right)\right]^{2}\right\}\right]}
$$

and

$$
\mathcal{P}_{x}(w)=\frac{\zeta_{0}^{3} x}{2 \pi v_{0}^{2} R} \int_{-\infty}^{+\infty} d v \int_{v^{\prime} / v_{0}}^{v / v_{0}+x / R} d \eta \frac{\exp \left\{-\frac{1}{2} \zeta_{0}^{3 / 2}\left[\left(\frac{v}{v_{0}}+\frac{x}{R}\right)^{2}+\left(\frac{v+w}{v_{0}}\right)^{2}\right]\right\}}{\left[1+\zeta_{0}^{3 / 4} \frac{x}{R} \int_{0}^{\infty} \frac{d \xi}{\sqrt{2 \pi}} \xi \exp \left\{-\frac{1}{2}\left(\xi+\zeta_{0}^{3 / 4} \eta\right)^{2}\right\}\right]^{2}}
$$


Changing the integration variables: $v=-\frac{x}{R} v_{0}+\zeta_{0}^{-3 / 4} v_{0} s$ and $\eta=(v+w) / v_{0}+\zeta_{0}^{-3 / 4} z$, and introducing rescaled (dimensionless) distance

$$
r \equiv \zeta_{0}^{3 / 4} \frac{x}{R}
$$

and rescaled (dimensionless) velocity difference

$$
\omega \equiv \zeta_{0}^{3 / 4} \frac{w}{v_{0}}=\zeta_{0}^{3 / 4} \frac{\left(v^{\prime}-v\right)}{v_{0}}
$$

for the corresponding probability density function $P_{r}(\omega)$ we obtain the following final result:

$$
P_{r}(\omega)=p_{0}(r) \delta(\omega-r)+\mathcal{P}_{r}(\omega) \theta(r-\omega)
$$

where

$$
p_{0}(r)=\int_{-\infty}^{+\infty} \frac{d s}{\sqrt{2 \pi}} \frac{\exp \left\{-\frac{1}{2} s^{2}\right\}}{\left[1+r \int_{0}^{\infty} \frac{d \xi}{\sqrt{2 \pi}} \xi \exp \left\{-\frac{1}{2}(\xi+s)^{2}\right\}\right]}
$$

and

$$
\mathcal{P}_{r}(\omega)=r \int_{-\infty}^{+\infty} \frac{d s}{\sqrt{2 \pi}} \int_{0}^{r-\omega} \frac{d z}{\sqrt{2 \pi}} \frac{\exp \left\{-\frac{1}{2} s^{2}-\frac{1}{2}(s+\omega-r)^{2}\right\}}{\left[1+r \int_{0}^{\infty} \frac{d \xi}{\sqrt{2 \pi}} \xi \exp \left\{-\frac{1}{2}(\xi+z+s+\omega-r)^{2}\right\}\right]^{2}}
$$

It is evident that this function is positively defined and it can be easily checked that for any value of $r$ it is normalized:

$$
\int_{-\infty}^{+\infty} d \omega P_{r}(\omega)=1
$$

We see that the distribution function $P_{r}(\omega)$ has rather specific structure (see Fig.4). According to eq. (120) for a given

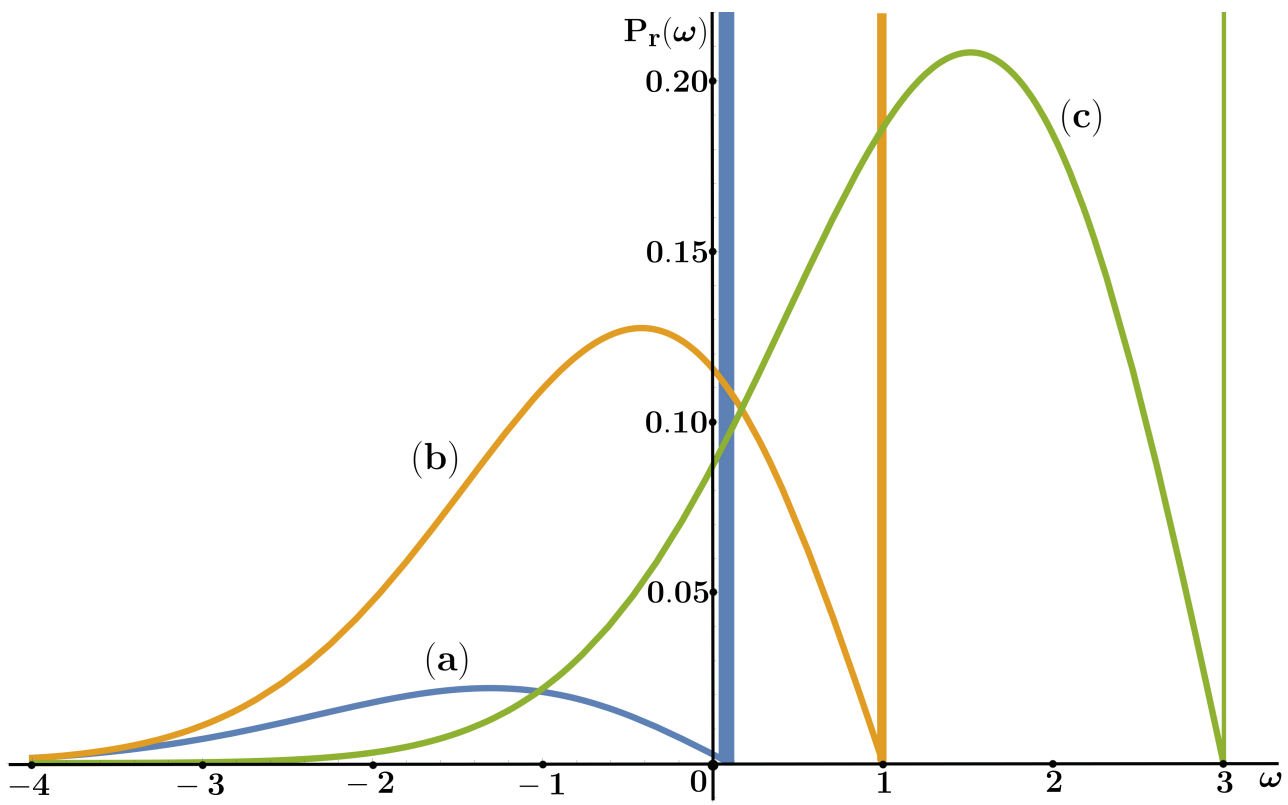

FIG. 4: Probability density function $P_{r}(\omega)$, eqs.(120)-(122) for: (a) $r=0.1$; (b) $r=1$; (c) $r=3$. The vertical lines at $\omega=r$ represent the $\delta$-functions, and the difference in the thickness of these lines symbolizes the relative values of the corresponding weights $p_{0}(r)$, eq.(121), which decrease with increasing $r$.

(rescaled) distance $r$ possible values of the (rescaled) velocity difference $\omega$ are bounded from above: $\omega \leq r$, or in terms 
of the original values, $\left(v^{\prime}-v\right) \leq \frac{x}{R} v_{0}$ In other words, at a given distance $x$ between two points at which we measure two velocities $v$ and $v^{\prime}$, their difference can not be bigger then $v_{0} x / R$, where, according to eq.(110), $v_{0} \propto\left(u / R^{2}\right)^{1 / 3}$ is the typical flow velocity at the injection scale $R$ of the random force of the strength $u$. Moreover, at $\omega=r$ (or at $\left.\left(v^{\prime}-v\right)=\frac{x}{R} v_{0}\right)$ the distribution function exhibits the $\delta$-function singularity.

Let us investigate the statistical properties of the velocity difference at small distances, $x \ll R$, or $r \ll 1$. In the limit of small values of the parameter $r$, the probability density function $P_{r}(\omega)$, eqs. (120)-(122), takes much more simple form:

$$
P_{r}(\omega) \simeq\left(1-\frac{1}{\sqrt{\pi}} r\right) \delta(\omega-r)+\frac{1}{\sqrt{\pi}} r(r-\omega) \exp \left\{-\frac{1}{2}(r-\omega)^{2}\right\} \theta(r-\omega)
$$

For even moments of the velocity difference $\left\langle\omega^{2 n}\right\rangle$ we find:

$$
\left\langle\omega^{2 n}\right\rangle=\int_{-\infty}^{+\infty} d \omega \omega^{2 n} P_{r}(\omega) \simeq r^{2 n}+C(n) r
$$

where $C(n)=2^{2 n+1} \Gamma(1+n)$. Then, the analytic continuation of the above result for arbitrary real values of the parameter $2 n \rightarrow q$, in the limit $r \ll 1$ yields:

$$
\left\langle\omega^{q}\right\rangle \simeq r^{q}+C(q / 2) r \simeq\left\{\begin{array}{l}
r^{q}, \text { for } q \leq 1 \\
C(q / 2) r, \text { for } q>1
\end{array}\right.
$$

Finally, introducing the exponent $\zeta(q)$ according to the definition $\left\langle\omega^{q}\right\rangle=r^{\zeta(q)}$ we recover the typical strong intermittency behavior [3] (see Fig.1):

$$
\zeta(q) \simeq\left\{\begin{array}{l}
q, \text { for } q \leq 1 \\
1, \text { for } q>1
\end{array}\right.
$$

\section{CONCLUSIONS}

In this paper we studied the statistical properties of of the velocity field $v(x, t)$ in the one-dimensional randomly forced Burgers turbulence, eq.(1). This system is known to be equivalent to the model of directed polymers in a random potential, eqs. (4)-(7), such that the the viscosity parameter $\nu$ in the Burger's equation is proportional to the temperature in the directed polymer system, $\nu=\frac{1}{2} T$, and the velocity $v(x, t)$ in the Burger's equation is the negative spatial derivative of the free energy $F(x, t)$ of the directed polymers. The parameter which characterizes the level of turbulence of the velocity field in the Burgers problem is the Reynolds number Re which in terms of the directed polymers notations is expressed as $R e=2(u R)^{1 / 3} / T$ where $R$ and $u$ are the correlation length and the strength of the random potential, eqs.(5) -(6) . Thus the strong turbulence regime where $R e \rightarrow \infty$ corresponds to the zero-temperature limit in the directed polymers system. In this limit in terms of the replica technique a general expression for the joint distribution function of two velocities $v(-x / 2, t)$ and $v(x / 2, t)$ separated by a finite distance $x$ has been derived, eqs.(111)-(113). Besides we have obtained an explicit expression for the probability density function for the corresponding velocity increment $w=v(-x / 2, t)-v(x / 2, t)$, eqs. (120)-(122), which was shown to exhibit rather specific structure. Namely, for any given distance $x$ the values of the velocity increment $w$ are bounded from above: $w \leq \frac{x}{R} v_{0}$, where $v_{0} \propto\left(u / R^{2}\right)^{1 / 3}$ is the typical flow velocity at the injection scale $R$ of the random potential. Moreover, at $w=\frac{x}{R} v_{0}$ the distribution function exhibits the $\delta$-function singularity which means that at a given distance $x$ the difference of two velocities $w=v(-x / 2, t)-v(x / 2, t)$ has a finite probability to be equal to $\frac{x}{R} v_{0}$. Using this distribution function at length scales much smaller than the injection length of the random potential, $x \ll R$ we have computed the moments of the velocity increment $\left\langle\omega^{q}\right\rangle$, eq.(126). Introducing the exponent $\zeta(q)$ according to the definition $\left\langle\omega^{q}\right\rangle \simeq r^{\zeta(q)}$ we have demonstrated that the function $\zeta(q)$ exhibits the behavior typical for strong intermittency phenomena, eq.(127), Fig.1.

Finally a few remarks about the status of the obtained results. First of all, as the considerations has been performed in the framework of the heuristic replica method, the proposed derivation can be considered as rigorous. Moreover, at the moment it is also difficult to say whether the obtained results are exact or not: on one hand, no approximations have been used in the performed calculations, but on the other hand, the considered derivation is based on the unproved crucial assumption about the vector replica symmetry breaking structure of the $N$-particle bosonic wave function in the zero-temperature limit which, in particular, contains undefined numerical factor $\zeta_{0}$, eq. (57) ([17], Section III). All that means that further more systematic study of the considered problem. is required. 


\section{Acknowledgments}

I am grateful to Kostya Khanin for numerous useful discussions.

I would like to thank the mathematical research institute MATRIX in Australia where part of this research was performed.

\section{Appendix A}

In this technical Appendix the derivatives of the functions $S\left(f_{1}, f_{2}, f_{3}\right)$ and $G\left(f_{1}, f_{2}, f_{3}\right)$, eqs. (99) and (100), will be calculated in the limit $\epsilon \rightarrow 0$. Redefining:

$$
\begin{aligned}
\xi & =\frac{1}{\gamma x} \tilde{\xi} \\
\epsilon & =\tilde{\epsilon} x
\end{aligned}
$$

and introducing velocities $v$ and $v^{\prime}$ instead of $f_{1}$ and $f_{3}$,

$$
\begin{aligned}
& f_{1}=-\tilde{\epsilon} x v+f_{2} \\
& f_{3}=-\tilde{\epsilon} x v^{\prime}
\end{aligned}
$$

we get

$$
\begin{aligned}
\frac{\partial}{\partial f_{1}} & =-\frac{1}{\tilde{\epsilon} x} \frac{\partial}{\partial v} \\
\frac{\partial}{\partial f_{3}} & =-\frac{1}{\tilde{\epsilon} x} \frac{\partial}{\partial v^{\prime}}
\end{aligned}
$$

According to eqs.(72), (63), (57) and (49), in the first order in $\epsilon \rightarrow 0$ we have:

$$
\begin{aligned}
& f_{01}=\frac{1}{2}(\beta m) \gamma^{2} x^{2}=\frac{1}{2} \sqrt{\zeta_{0}} T_{*}\left(\frac{x}{R}\right)^{2} \equiv f_{0} \\
& f_{01}=\frac{1}{2}(\beta m) \gamma^{2}(x-\epsilon)^{2} \simeq f_{0}+2 \tilde{\epsilon} f_{0} \\
& f_{03}=\frac{1}{2}(\beta m) \gamma^{2} \epsilon^{2} \rightarrow 0
\end{aligned}
$$

Substituting eqs.(A.1)-(A.9) into eqs.(99) and (100) we find

$$
\begin{aligned}
S\left(v, f_{2}, v^{\prime}\right) & =\frac{\zeta_{0}}{T_{*} \gamma x} \int_{0}^{+\infty} d y \int_{-\infty}^{+\infty} \frac{d \tilde{\xi}}{\sqrt{2 \pi}} \exp \left\{-\frac{\tilde{\xi}^{2}}{2 \gamma^{2} x^{2}}\right\} \times \\
& \times\left[1-\theta\left(y+f_{0}-f_{2}+\tilde{\epsilon} x v-\tilde{\xi}\right) \theta\left(y+f_{0}-f_{2}-2 \tilde{\epsilon} f_{0}+\tilde{\epsilon} \tilde{\xi}-\tilde{\xi}\right) \theta\left(y+\tilde{\epsilon} x v^{\prime}-\tilde{\epsilon} \tilde{\xi}\right)\right]
\end{aligned}
$$

and

$$
G\left(v, f_{2}, v^{\prime}\right)=\frac{1}{\gamma x} \int_{-\infty}^{+\infty} \frac{d \tilde{\xi}}{\sqrt{2 \pi}} \exp \left\{-\frac{\tilde{\xi}^{2}}{2 \gamma^{2} x^{2}}\right\}\left[1-\theta\left(f_{0}-f_{2}+\tilde{\epsilon} x v-\tilde{\xi}\right) \theta\left(f_{0}-f_{2}-2 \tilde{\epsilon} f_{0}+\tilde{\epsilon} \tilde{\xi}-\tilde{\xi}\right) \theta\left(\tilde{\epsilon} x v^{\prime}-\tilde{\epsilon} \tilde{\xi}\right)\right]
$$

The calculation of the derivatives of these functions is straightforward. For example, for the derivative $G_{1}^{\prime} \equiv-\frac{1}{\tilde{\epsilon} x} \frac{\partial}{\partial v} G$ we get

$$
G_{1}^{\prime}=\frac{1}{\gamma x} \int_{-\infty}^{+\infty} \frac{d \tilde{\xi}}{\sqrt{2 \pi}} \exp \left\{-\frac{\tilde{\xi}^{2}}{2 \gamma^{2} x^{2}}\right\} \delta\left(f_{0}-f_{2}+\tilde{\epsilon} x v-\tilde{\xi}\right) \theta\left(f_{0}-f_{2}-2 \tilde{\epsilon} f_{0}+\tilde{\epsilon} \tilde{\xi}-\tilde{\xi}\right) \theta\left(\tilde{\epsilon} x v^{\prime}-\tilde{\epsilon} \tilde{\xi}\right)
$$


Taking the limit $\epsilon \rightarrow 0$ we find:

$$
\lim _{\epsilon \rightarrow 0} G_{1}^{\prime}=\frac{1}{\gamma x \sqrt{2 \pi}} \exp \left\{-\frac{\left(f_{2}-f_{0}\right)^{2}}{2 \gamma^{2} x^{2}}\right\} \theta\left(f_{2}-f_{0}+x v^{\prime}\right) \theta\left(-f_{0}-f_{2}-x v\right)
$$

In a similar way we obtain the rest of the derivatives:

$$
\begin{aligned}
& \lim _{\epsilon \rightarrow 0} G_{2}^{\prime}=\frac{1}{\gamma x \sqrt{2 \pi}} \exp \left\{-\frac{\left(f_{2}-f_{0}\right)^{2}}{2 \gamma^{2} x^{2}}\right\} \theta\left(f_{2}-f_{0}+x v^{\prime}\right) \theta\left(f_{0}+f_{2}+x v\right) \\
& \lim _{\epsilon \rightarrow 0} G_{3}^{\prime}=\frac{1}{\gamma \epsilon \sqrt{2 \pi}} \exp \left\{-\frac{\left(v^{\prime}\right)^{2}}{2 \gamma^{2} x^{2}}\right\} \theta\left(f_{0}-f_{2}-x v^{\prime}\right) \\
& \lim _{\epsilon \rightarrow 0} G_{12}^{\prime \prime}=-\frac{1}{\gamma \epsilon \sqrt{2 \pi}} \exp \left\{-\frac{\left(x v+2 f_{0}\right)^{2}}{2 \gamma^{2} x^{2}}\right\} \theta\left(x v^{\prime}-x v-2 f_{0}\right) \delta\left(f_{2}+f_{0}+x v\right) \\
& \lim _{\epsilon \rightarrow 0} G_{13}^{\prime \prime}=-\frac{1}{\gamma \epsilon \sqrt{2 \pi}} \exp \left\{-\frac{\left(v^{\prime}\right)^{2}}{2 \gamma^{2} x^{2}}\right\} \theta\left(x v^{\prime}-x v-2 f_{0}\right) \delta\left(f_{2}-f_{0}+x v^{\prime}\right) \\
& \lim _{\epsilon \rightarrow 0} G_{13}^{\prime \prime}=-\frac{1}{\gamma \epsilon \sqrt{2 \pi}} \exp \left\{-\frac{\left(v^{\prime}\right)^{2}}{2 \gamma^{2} x^{2}}\right\} \theta\left(x v-x v^{\prime}+2 f_{0}\right) \delta\left(f_{2}-f_{0}+x v^{\prime}\right) \\
& \lim _{\epsilon \rightarrow 0} G_{123}^{\prime \prime \prime}=\frac{1}{\gamma \epsilon^{2} \sqrt{2 \pi}} \exp \left\{-\frac{\left(v^{\prime}\right)^{2}}{2 \gamma^{2} x^{2}}\right\} \delta\left(x v^{\prime}-x v+2 f_{0}\right) \delta\left(f_{2}-f_{0}+x v^{\prime}\right) \\
& \lim _{\epsilon \rightarrow 0} S_{1}^{\prime}=\frac{\zeta_{0}}{T_{*} \gamma x \sqrt{2 \pi}} \int_{0}^{\infty} d y \exp \left\{-\frac{\left(y-f_{2}+f_{0}\right)^{2}}{2 \gamma^{2} x^{2}}\right\} \theta\left(y-f_{2}-f_{0}-x v\right) \\
& \lim _{\epsilon \rightarrow 0} S_{2}^{\prime}=\frac{\zeta_{0}}{T_{*} \gamma x \sqrt{2 \pi}} \int_{0}^{\infty} d y \exp \left\{-\frac{\left(y-f_{2}+f_{0}\right)^{2}}{2 \gamma^{2} x^{2}}\right\} \theta\left(f_{2}+f_{0}+x v-y\right) \\
& \lim _{\epsilon \rightarrow 0} S_{3}^{\prime}=\frac{\zeta_{0}}{T_{*} \gamma x \sqrt{2 \pi}} \int_{0}^{\infty} d y \exp \left\{-\frac{\left(y+x v^{\prime}\right)^{2}}{2 \gamma^{2} x^{2}}\right\} \theta\left(f_{0}-f_{2}-x v^{\prime}-y\right) \theta\left(f_{0}-f_{2}-x v^{\prime}\right) \\
& \lim _{\epsilon \rightarrow 0} S_{12}^{\prime \prime}=-\frac{\zeta_{0}}{T_{*} \gamma \epsilon \sqrt{2 \pi}} \exp \left\{-\frac{\left(x v+2 f_{0}\right)^{2}}{2 \gamma^{2} x^{2}}\right\} \theta\left(x v+f_{0}+f_{2}\right) \\
& \lim _{\epsilon \rightarrow 0} S_{13}^{\prime \prime}=-\frac{\zeta_{0}}{T_{*} \gamma x \sqrt{2 \pi}} \exp \left\{-\frac{\left(f_{2}-f_{0}\right)^{2}}{2 \gamma^{2} x^{2}}\right\} \theta\left(-f_{2}-f_{0}-x v\right) \theta\left(f_{0}-f_{2}-x v^{\prime}\right) \\
& \lim _{\epsilon \rightarrow 0} S_{23}^{\prime \prime}=-\frac{\zeta_{0}}{T_{*} \gamma x \sqrt{2 \pi}} \exp \left\{-\frac{\left(f_{2}-f_{0}\right)^{2}}{2 \gamma^{2} x^{2}}\right\} \theta\left(f_{2}+f_{0}+x v\right) \theta\left(f_{0}-f_{2}-x v^{\prime}\right) \\
& \lim _{\epsilon \rightarrow 0} S_{123}^{\prime \prime \prime}=\frac{\zeta_{0}}{T_{*} \gamma \epsilon \sqrt{2 \pi}} \exp \left\{-\frac{\left(x v+2 f_{0}\right)^{2}}{2 \gamma^{2} x^{2}}\right\} \theta\left(x v+2 f_{0}-x v^{\prime}\right)
\end{aligned}
$$

We see that in the limit $\epsilon \rightarrow 0$ according to the above expressions, eqs. A.13 - A.26), the only non-zero contribution to the function $P_{x}\left(v, v^{\prime}\right)$ in eqs.(101)-(102) are given by the two terms: $G_{123}^{\prime \prime \prime} \propto 1 / \epsilon^{2}$, eq. (A.19), and the product $G_{3}^{\prime} S_{12}^{\prime \prime} \propto 1 / \epsilon^{2}$, eqs. A.15) and A.23.

[1] J.M. Burgers, The Nonlinear Diffusion Equation (Reidel, Dordrecht, (1974)).

[2] Ya. G. Sinai, Commun.Math.Phys. 148, 601 (1992); J.Stat.Phys. 64, 1 (1991)

[3] J.P.Bouchaud, M.Mezard and G.Parisi, Phys.Rev. E 52, 3656 (1995)

[4] J.Bec and K.Khanin, Physics Reports 447, 1 (2007)

[5] A. Kolmogorov, C. R. Acad. Sci. USSR, 30, 301 (1941); 32, 16 (1941)

[6] A. Kolmogorov, J Fluid Mech, 13, 82 (1962)

[7] A.M.Obukhov, J Fluid Mech, 13, 77 (1962) 
[8] U.Frisch, P.L.Sulem and M.Nelkin, J Fluid Mech, 87, 719 (1978)

[9] F.Argoul et al, Nature, 338, 51 (1989)

[10] B.Castaing, Y.Gagne and E.J.Hopfilger, Physica D, 46, 177 (1990)

[11] R.Benzi et al, Phys. Rev. E48, R29 (1993)

[12] Zhen-Su She and E.Leveque, Phys Rev. Lett., 72, 336 (1994)

[13] B.Dubrulle, Phys Rev. Lett., 73, 959 (1994)

[14] A.Arneodo, E.Bacry and J.F.Muzy, Physica A, 213, 232 (1995)

[15] M.Kardar, G.Parisi, Y-C.Zhang, Phys. Rev. Lett. 56, 889 (1986)

[16] T. Halpin-Healy and Y-C. Zhang, Phys. Rep. 254, 215 (1995).

[17] V.Dotsenko, J. Stat. Mech. 123304 (2016)

[18] V.Dotsenko, J. Stat. Mech. P02016 (2015)

[19] V. Dotsenko, Statistical properties of one-dimensional directed polymers in a random potential, arXiv:1703.04305; in Order, Disorder and Criticality, volume 5, World Scientific, 2018

[20] Lieb E H and Liniger W (1963) Phys. Rev. 130, 1605

[21] McGuire J B (1964) J. Math. Phys. 5, 622

[22] Yang C N (1968) Phys. Rev. 168, 1920

[23] E.Agoritsas, S.Bustingorry, V.Lecomte, G.Schehr and T.Giamarchi (2012) Phys. Rev. E 86, 031144; E.Agoritsas, V.Lecomte and T.Giamarchi (2013) Phys. Rev. E 87, 042406; E.Agoritsas, V.Lecomte and T.Giamarchi (2013) Phys. Rev. E 87, 062405 